\title{
Managed Care Perspective on Three New Agents for Type 2 Diabetes
}

\author{
Shawna VanDeKoppel, PharmD; Hae Mi Choe, PharmD, CDE; and Burgunda V. Sweet, PharmD, FASHP
}

\begin{abstract}
BACKGROUND: Despite effective monotherapy for diabetes, approximately $50 \%$ of patients require additional medications after 3 years to achieve target glycosylated hemoglobin (A1C) $<7 \%$. Three new agents, each the first in its therapeutic class with a unique mechanism of action, have been approved for the treatment of type 2 diabetes by the U.S. Food and Drug Administration: pramlintide in March 2005, exenatide in April 2005, and sitagliptin in October 2006.
\end{abstract}

OBJECTIVE: To review the efficacy and safety of 3 new agents for type 2 diabetes (exenatide and pramlintide by subcutaneous injection and sitagliptin by oral administration) and to define their place in therapy given their relatively high cost and unknown long-term safety and efficacy.

METHODS: A MEDLINE search (1950 to June 2007) for English-language articles of studies in human subjects was conducted using these search terms: type 2 diabetes, exenatide, pramlintide, and sitagliptin. This database was supplemented by systematic reviews and meta-analyses through December 2007 and reference citations from the articles identified in the MEDLINE search.

RESULTS: Exenatide, pramlintide, and sitagliptin have all been shown to have a modest effect on reducing A1C. In several relatively short-term trials (generally 15-30 weeks in duration), exenatide injection has been shown to be safe and effective for patients with type 2 diabetes who are either at the maximum doses of or cannot tolerate metformin, sulfonylurea, and/or thiazolidinedione therapy and need to further decrease A1C by at least $0.5 \%$ to $1 \%$. While weight loss of $1.5 \mathrm{~kg}$ to $2.5 \mathrm{~kg}$ associated with exenatide is modest, this effect is of obvious value in many patients with type 2 diabetes. Nausea is the most notable side effect with exenatide, occurring in up to $50 \%$ of patients within the first 8 weeks of therapy but decreasing to $5 \%$ to $10 \%$ by week 24 . In addition, the risk for hypoglycemia increases 4- to 5-fold when used in combination with sulfonylureas. Like exenatide, pramlintide injection reduces $\mathrm{A} 1 \mathrm{C}$ by approximately $0.5 \%$ to $1 \%$, carries the advantage of modest weight loss (1.5 kg over 1 year), and has a high incidence of nausea. Pramlintide can also result in severe hypoglycemia because of its ability to enhance the effects of insulin, a concern given that it is only indicated for use in combination with insulin. Sitagliptin is an oral agent that can be used alone or in combination with other oral hypoglycemic agents and has been shown to reduce $\mathrm{A} 1 \mathrm{C}$ by $0.5 \%$ to $0.7 \%$. It has only been studied in short-term studies, to date, so the long-term safety and efficacy are unknown. There is potential for severe allergic and dermatologic reactions with sitagliptin.

CONCLUSIONS: The 3 new agents for the management of type 2 diabetes have been shown to reduce $\mathrm{A} 1 \mathrm{C}$ by no more than $1.0 \%$, modest by comparison with insulin and the older oral agents. The 3 newer agents have either modest positive effects on body weight or are weight neutral. The longterm safety and efficacy of the 3 newer agents are unknown, and their cost is considerably higher than the first-line agents, metformin and sufonylureas, which are available by generic name. The newer agents offer treatment options in select patients, although their use should be reserved for patients who are not adequately managed by agents with known longterm efficacy and safety, which are often available at a lower cost.

J Manag Care Pharm. 2008;14(4):363-80

Copyright $\odot$ 2008, Academy of Managed Care Pharmacy. All rights reserved.

\section{What is already known about this subject}

- The 2006 consensus statement from the American Diabetes Association (ADA) provides an overall summary for the management of hyperglycemia in patients with type 2 diabetes. Exenatide and pramlintide are briefly mentioned in this document; sitagliptin is not mentioned at all. None of these 3 agents is included in the ADA treatment algorithm to define their place in therapy.

- The new Standards of Medical Care in Diabetes-2008 do not elaborate on the place in therapy for these 3 newer agents. There is a brief mention of pharmacologic therapy, with only limited data on the new agents (stating that the new agents may be appropriate in some patients) and noting the more recent (2007-2008) safety concerns with the thiazolidinediones since the consensus guidelines were developed.

- The American Association of Clinical Endocrinologists Medical Guidelines for Clinical Practice for the Management of Diabetes Mellitus (2007) dedicate 1 to 2 short summary paragraphs reviewing the new agents. This guideline provides all viable options for monotherapy and combination therapy but does not provide guidance on place in therapy, overall treatment algorithm, or how the new agents might fit within a hierarchical treatment algorithm.

\section{What this study adds}

- There are no long-term safety and efficacy data for any of these 3 newer agents, and given their relatively high cost, the place in therapy is only in patients with diabetes who are not willing or able to tolerate the standard first-line treatment options.

- The ability of drug companies to influence prescribing through direct and indirect marketing strategies can make it difficult for physicians and patients to objectively assess the benefits and risks of the new agents. This comprehensive review of the 3 newer agents and presentation of current (2008) real-world prices for all of the drugs in the treatment guidelines may help clarify their place in therapy.

I t is estimated that approximately 20.8 million Americans have type 2 diabetes. ${ }^{1}$ More than 90\% of all newly diagnosed cases of diabetes are patients with type 2 disease. Because this number continues to grow each year, significant time and money has been spent to better understand the disease pathology and to discover new pharmacological treatments. The purpose of this paper is to briefly review the pathophysiology of type 2 
diabetes, to summarize the data for agents (exenatide, pramlintide, and sitagliptin) that have recently become available, and to help the clinician identify clinical situations in which the new agents should be considered in the treatment algorithm.

\section{Glucose Homeostasis and Pathophysiology of Type 2 Diabetes}

Normal glucose metabolism involves a balancing act between insulin and glucagon. When glucose is consumed, beta cells in the pancreas are stimulated to release insulin and suppress glucagon. Insulin is typically secreted in 2 phases: a quick, immediate increase in response to glucose intake followed by a slower, sustained release. Insulin allows for cellular uptake of glucose, the energy source for the cell. It also inhibits any cellular production of glucose. ${ }^{2}$ Glucagon, another regulatory hormone, reacts in response to low plasma glucose levels. It stimulates the production of glucose via gluconeogenesis, ketogenesis, and hepatic breakdown of glycogen.

Other hormones have also been found to play key roles in regulating glucose homeostasis. Incretin hormones are released from the gut after a meal. One of these incretin hormones, glucagon-like peptide-1 (GLP-1), binds to the beta-cell membrane in the pancreas, thereby stimulating insulin secretion by the beta cell. GLP-1 is not activated when glucose concentrations are below a certain threshold, thereby preventing glucose levels from becoming too low. Studies have shown that GLP-1 also appears to increase cell glucose sensitivity and aids in insulin synthesis and beta-cell function. ${ }^{3-6}$

Amylin, a neuroendocrine hormone, has also been found to be important for glucose metabolism. Amylin is released from the beta cells of the pancreas in conjunction with insulin secretion. It binds to receptors in the brain to aid in the regulation of glucose by inhibiting glucagon secretion. This allows the body to use glucose recently ingested instead of glucose via gluconeogenesis. ${ }^{7-9}$

In type 2 diabetes, regulation of glucose utilization is impaired because of decreased pancreatic beta-cell function, which causes decreased insulin and amylin secretion. The result is decreased blood glucose utilization and over-expression of glucagon. Unopposed glucagon will subsequently increase glucose production from endogenous sources and promote glycogen breakdown. Incretin hormone secretion, including GLP-1, is also decreased in type 2 diabetes, further impeding insulin production by and secretion from beta cells. The net result is hyperglycemia and perpetuation of the unregulated cycle., 2,3,9

\section{Management of Type 2 Diabetes}

In order to prevent the complications of diabetes, the American Diabetes Association (ADA) recommends glycosylated hemoglobin $(\mathrm{AlC})<7 \%$. Lifestyle interventions, such as good nutrition and exercise, are an important component of managing type 2 diabetes. However, for many patients, diet and exercise alone are inadequate to maintain optimal blood glucose control. As such, the current ADA guidelines recommend initiation of metformin at the time of diagnosis and consider metformin to be the only drug for diabetes prevention. ${ }^{10}$

The ADA and the American Association of Clinical Endocrinologists (AACE) provide an overview of the treatment options for glycemic management. ${ }^{10,11}$ The different classes of agents available for the management of diabetes are shown in Table 1. Treatment is usually initiated with oral metformin monotherapy; a sulfonylurea should be considered in patients in whom metformin is not an option (i.e., those with reduced renal function)..$^{10-14}$ Despite optimal monotherapy, approximately 50\% of patients with diabetes will require additional medications after 3 years to achieve an $\mathrm{AlC}<7 \% .{ }^{14} \mathrm{~A}$ second oral agent is often added at this point. If a patient is receiving the maximum dose of metformin, a sulfonylurea is typically added..$^{10,12,14,15}$ If a patient is unable to tolerate or has a contraindication to metformin or sulfonylurea therapy, a thiazolidinedione or bedtime insulin may be added to aid in further reducing AlC..$^{10,12,16,17}$ Insulin may be favored over a thiazolidinedione if levels of glycemia are high $(\geq 8.5 \%)$ or if a patient cannot tolerate a thiazolidinedione. Despite the variety of treatment options available, combination therapy with or without insulin may still be inadequate to achieve glycemic control.

Bolen et al. conducted a systematic review outlining the comparative efficacy and safety of the oral medications (excluding sitagliptin) for type 2 diabetes. ${ }^{18}$ In recent years, compounds that mimic the actions of the natural endocrine hormones GLP-1 and amylin have been isolated and are now available for the treatment of type 2 diabetes. Two of these agents, exenatide and pramlintide, are available for subcutaneous use only. Sitagliptin is a new oral agent that inhibits the metabolism of GLP-1. The availability of new agents with differing mechanisms of action for a disease state whose incidence has increased by $54 \%$ in the past 7 years in the United States is generally viewed as favorable. However, there are concerns with the routine use of newer agents given the lack of long-term efficacy data, their high cost, and ongoing reports through post-marketing data regarding overall safety. ${ }^{19}$

\section{Clinical Studies \\ Exenatide}

Exenatide (Byetta, Amylin Pharmaceuticals), is an incretin mimetic similar to GLP-1 that was originally discovered in Gila monster saliva. Exenatide was approved by the U.S. Food and Drug Administration (FDA) in April 2005 and is labeled for adjunctive therapy to improve glycemic control in patients with type 2 diabetes who are taking metformin, a sulfonylurea, or a thiazolidinedione, either alone or in combination. ${ }^{20}$ Exenatide binds to the GLP-1 receptor in the gut, but it has increased potency and a longer duration of action than endogenous GLP-1.20-22 It has been shown to potentiate insulin secretion, decrease glucagon secretion, decrease gastric emptying time, and enhance satiety. 
(TABLE 1) Pharmacological Agents Used for the Treatment of Type 2 Diabetes in Order of Effect on A1C 10,19,55

\begin{tabular}{|c|c|c|c|c|c|c|}
\hline $\begin{array}{l}\text { Drug } \\
\text { Class }\end{array}$ & $\begin{array}{l}\text { Mechanism } \\
\text { of Action }\end{array}$ & $\begin{array}{c}\% \text { AlC } \\
\text { Reduction }\end{array}$ & Medication & $\begin{array}{c}\text { Dosage } \\
\text { Form }\end{array}$ & $\begin{array}{l}\text { Usual } \\
\text { Dose }\end{array}$ & $\begin{array}{c}\text { Drug Cost (\$) } \\
\text { (per month) }\end{array}$ \\
\hline Insulins & - Provides exogenous insulin & $>2.5 \%$ & $\begin{array}{l}\text { Insulin glargine } \\
\text { Insulin lispro } \\
\text { Insulin human isophane }\end{array}$ & $100 \mathrm{u} / \mathrm{mL}, 10 \mathrm{~mL}$ vials & Various & $\begin{array}{l}135 \\
150-225 \\
90-120 \\
\end{array}$ \\
\hline Biguanides & $\begin{array}{l}\text { - Decreases endogenous glucose } \\
\text { synthesis } \\
\text { - Decreases intestinal absorption } \\
\text { of glucose } \\
\text { - Increases insulin sensitivity }\end{array}$ & $1.5 \%$ & $\begin{array}{l}\text { Metformin } \\
\text { Metformin ER }\end{array}$ & $\begin{array}{l}\text { 500/750/850/1,000 mg } \\
\text { tablets } \\
500 / 750 / 1,000 \mathrm{mg} \text { tablets }\end{array}$ & $\begin{array}{l}1,000 \mathrm{mg} \text { BID } \\
1,000 \mathrm{mg} \text { daily }\end{array}$ & 29 \\
\hline Sulfonylureas & - Stimulates insulin release & $1.5 \%$ & $\begin{array}{l}\text { Glipizide } \\
\text { Glipizide XL } \\
\text { Glimepiride } \\
\text { Glyburide }\end{array}$ & $\begin{array}{l}\text { 5/10 mg tablets } \\
2.5 / 5 / 10 \mathrm{mg} \text { tablets } \\
1 / 2 / 4 \mathrm{mg} \text { tablets } \\
1.25 / 2.5 / 5 \mathrm{mg} \text { tablets }\end{array}$ & $\begin{array}{l}5 \mathrm{mg} \text { BID } \\
5 \mathrm{mg} \text { daily } \\
2 \mathrm{mg} \text { daily } \\
5 \mathrm{mg} \text { daily }\end{array}$ & $\begin{array}{l}10 \\
11 \\
9 \\
12\end{array}$ \\
\hline Glinides & - Stimulates insulin release & $1.0 \%-1.5 \%$ & $\begin{array}{l}\text { Nateglinide } \\
\text { Repaglinide }\end{array}$ & $\begin{array}{l}60 / 120 \mathrm{mg} \text { tablets } \\
0.5 / 1 / 2 \mathrm{mg} \text { tablets }\end{array}$ & $\begin{array}{l}60-120 \mathrm{mg} \mathrm{AC} \\
0.5-4 \mathrm{mg} \mathrm{AC}\end{array}$ & $\begin{array}{l}125 \\
150-240\end{array}$ \\
\hline $\begin{array}{l}\text { Thiazolidine- } \\
\text { diones }\end{array}$ & - Decreases insulin resistance & $0.8 \%-1.0 \%$ & $\begin{array}{l}\text { Pioglitazone } \\
\text { Pioglitazone/metformin } \\
\text { Rosiglitazone } \\
\text { Rosiglitazone/metformin }\end{array}$ & $\begin{array}{l}\text { 15/30/45 mg tablets } \\
\text { 15/500; 15/850 mg tablets } \\
\text { 2/4/8 mg tablets } \\
\text { 2/500; 4/500; 2/1,000; } \\
\text { 4/1,000 mg tablets }\end{array}$ & $\begin{array}{l}30 \mathrm{mg} \text { daily } \\
1 \text { tablet BID } \\
4-8 \mathrm{mg} \text { daily } \\
1 \text { tablet BID }\end{array}$ & $\begin{array}{l}181 \\
192 \\
155 \\
130-226\end{array}$ \\
\hline $\begin{array}{l}\text { Incretin } \\
\text { mimetics }\end{array}$ & $\begin{array}{l}\text { - Potentiates insulin secretion } \\
\text { - Decreases glucagon secretion } \\
\text { - Decreases gastric emptying time } \\
\text { - Enhances satiety }\end{array}$ & $0.5 \%-1.0 \%$ & Exenatide & $\begin{array}{l}\text { Pre-filled subcutaneous } \\
\text { pen ( } 5 \mathrm{mcg} \text { and } 10 \mathrm{mcg} \text { ) }\end{array}$ & 5-10 mcg BID & $213-230$ \\
\hline $\begin{array}{l}\text { Amylino- } \\
\text { mimetics }\end{array}$ & $\begin{array}{l}\text { - Decreases post-prandial gluca- } \\
\text { gon secretion } \\
\text { - Slows gastric emptying } \\
\text { - Increases satiety }\end{array}$ & $0.5 \%-1.0 \%$ & Pramlintide & $\begin{array}{l}5 \mathrm{ml} \text { vial }(600 \mathrm{mcg} / \mathrm{mL}) \\
\text { Pre-filled subcutaneous } \\
\text { pen ( } 60 \mathrm{mcg} \text { and } 120 \mathrm{mcg})\end{array}$ & $\begin{array}{l}60-120 \mathrm{mcg} \\
\text { TID }\end{array}$ & $232-440$ \\
\hline $\begin{array}{l}\text { Alpha- } \\
\text { glycosidase } \\
\text { inhibitors }\end{array}$ & $\begin{array}{l}\text { - Decreases digestion of poly- } \\
\text { saccharides } \\
\text { - Decreases post-prandial glucose }\end{array}$ & $0.5 \%-0.8 \%$ & $\begin{array}{l}\text { Acarbose } \\
\text { Miglitol }\end{array}$ & $\begin{array}{l}\text { 25/50/100 mg tablets } \\
\text { 25/50/100 mg tablets }\end{array}$ & $\begin{array}{l}25 \mathrm{mg} \text { TID } \\
25 \mathrm{mg} \text { TID }\end{array}$ & $\begin{array}{l}85 \\
80\end{array}$ \\
\hline $\begin{array}{l}\text { DPP-4 } \\
\text { inhibitors }\end{array}$ & $\begin{array}{l}\text { - Inhibits incretin hormone } \\
\text { metabolism }\end{array}$ & $0.5 \%-0.9 \%$ & Sitagliptin & 25/50/100 mg tablets & 100 mg daily & 171 \\
\hline
\end{tabular}

Products available as a generic formulations are shown in bold.

Cost data from www.drugstore.com. Accessed April 19, 2008. 55

$A C=$ before meals; $B I D=$ twice daily; $D P P-4=$ dipeptidyl peptidase-4; $E R=$ extended release; $T I D=3$ times daily

It may also promote beta-cell synthesis and proliferation. ${ }^{20-23}$ In an in vitro trial using human pancreatic islet cells, Chen et al. demonstrated that exenatide reduced apoptotic factors and maintained beta-cell function. ${ }^{24}$ Fineman et al. also showed a possible improvement in beta-cell function with exenatide therapy. ${ }^{25}$

\section{Efficacy of Exenatide}

Several studies have been conducted that evaluated the safety and efficacy of exenatide. Overall, twice-daily subcutaneous dosing of exenatide was able to decrease $\mathrm{AlC}$ and fasting plasma glucose while also reducing weight in patients with type 2 diabetes.
These effects were initially shown in a 28-day trial comparing twice-daily administration of placebo or exenatide $2.5 \mathrm{mcg}$, $5 \mathrm{mcg}, 7.5 \mathrm{mcg}$, or $10 \mathrm{mcg} .{ }^{26}$ All exenatide treatment groups displayed significant decreases in $\mathrm{AlC}$, with the absolute decrease being dose-dependent. There was also a dose-related decrease in weight compared with baseline by day 28 with all exenatide doses compared with no change in weight in subjects receiving placebo. Only the exenatide $7.5 \mathrm{mcg}$ and $10 \mathrm{mcg}$ groups experienced significant weight loss compared with baseline $(-1.4 \mathrm{~kg}$ and $-1.7 \mathrm{~kg}$, respectively; $P<0.010)$. In another 28 -day trial, 109 patients were randomized to receive placebo or 1 of 
3 exenatide $0.08 \mathrm{mcg}$ per $\mathrm{kg}$ regimens (twice-daily, breakfast/ dinner; twice-daily, breakfast/bedtime; or 3-times-daily). ${ }^{25}$ There was a significant decrease in AlC compared with placebo for the twice-daily (breakfast/dinner) and 3-times-daily regimens $(-1.1 \%$ and $-1.0 \%$ versus $-0.3 \%, P<0.001)$ and for the twice-daily (breakfast/bedtime) regimen $(-0.7 \%$ versus $-0.3 \%, P=0.006)$. There was no difference in fasting plasma glucose, body weight, or lipid levels between any of the treatment groups.

Hypoglycemia occurred in 15\% of patients overall (active and placebo groups) and was reported to only be seen in patients who were also receiving a sulfonylurea. Unfortunately, no specific data on the incidence of hypoglycemia, either overall or in the subgroup of patients receiving a sulfonylurea, were reported. Nausea was the most commonly reported adverse effect, with exenatide therapy occurring in $31 \%$ of patients overall. However, this declined to $13 \%$ by day 28 . This study demonstrated that short-term therapy with exenatide is effective in combination with a sulfonylurea and/or metformin. However, it suggested that the risk for hypoglycemia is higher in patients receiving concomitant sulfonylurea therapy, although no specific data were provided to assess this risk. In addition, this study was limited by its short duration.

The long-term studies evaluating the safety and efficacy of exenatide therapy for type 2 diabetes are summarized in Table 2 . Buse et al. conducted a blinded, placebo-controlled, randomized study of 377 patients with type 2 diabetes who were not controlled on the maximum dose of a sulfonylurea. ${ }^{27}$ Baseline characteristics of all treatment groups were similar, with an average $\mathrm{AlC}$ of $8.6 \%$, mean age of 55 years, and an average body mass index (BMI) of $33 \mathrm{~kg}$ per $\mathrm{m}^{2}$. Patients were randomized to receive placebo or exenatide $5 \mathrm{mcg}$ or $10 \mathrm{mcg}$ subcutaneously twice daily for 30 weeks. All patients who were to receive exenatide were initiated at $5 \mathrm{mcg}$ twice daily for 4 weeks to improve tolerability. The high-dose group then had their dose increased to 10 mcg twice daily. Exenatide significantly decreased AlC in both treatment groups, with mean decreases of $0.46 \%$ and $0.86 \%$ in the $5 \mathrm{mcg}$ and $10 \mathrm{mcg}$ groups, respectively, compared with a $0.12 \%$ increase in the placebo group. There were no differences in fasting plasma glucose seen between exenatide $5 \mathrm{mcg}$ twice daily and placebo, but there was a significant decrease when exenatide $10 \mathrm{mcg}$ twice daily was compared with placebo $(-0.6 \mathrm{mmol}$ per $\mathrm{L}$ vs. $0.4 \mathrm{mmol}$ per $\mathrm{L}, \mathrm{P}<0.050$ ).

There was a reduction in the proinsulin:insulin ratio in the exenatide $10 \mathrm{mcg}$ group at week 30 when compared with placebo. There was also a significant decrease in fasting plasma insulin in patients receiving exenatide (both doses) versus those receiving placebo. At week 30, only the exenatide $10 \mathrm{mcg}$ group showed a significant weight loss (-1.6 kg) compared with placebo $(-0.6 \mathrm{~kg})$. Nausea, the most common side effect reported, was seen in $51 \%$ of those receiving exenatide $10 \mathrm{mcg}, 39 \%$ of those receiving exenatide $5 \mathrm{mcg}$, and $7 \%$ of those receiving placebo. Nausea was most prevalent within the first 4 to 8 weeks of therapy, with the incidence decreasing to $5 \%$ to $10 \%$ by week 24 of therapy. Mild to moderate hypoglycemia was reported at in 36\%, $14 \%$, and $3 \%$ of subjects in the $10 \mathrm{mcg}, 5 \mathrm{mcg}$, and placebo groups, respectively. Other common adverse events with exenatide therapy included dizziness, feeling jittery, vomiting, diarrhea, and upper respiratory infection. This study demonstrated that adding exenatide to patients not adequately controlled on sulfonylurea therapy can improve AlC control in patients with type 2 diabetes. Exenatide 10 mcg subcutaneously twice daily provided additional improvement in fasting plasma glucose, proinsulin:insulin ratio, and weight loss.

DeFronzo et al. conducted a similar blinded, placebocontrolled, randomized study in 336 patients with type 2 diabetes who were not adequately controlled on metformin (1,500 mg per day). ${ }^{28}$ All patients (treatment and placebo groups combined) had similar baseline characteristics (AlC 8.2\%, mean age 53 years, and average BMI $34 \mathrm{~kg} / \mathrm{m}^{2}$ ). Patients were randomized to receive placebo or exenatide ( $5 \mathrm{mcg}$ or $10 \mathrm{mcg}$ twice daily) for 30 weeks. Exenatide $5 \mathrm{mcg}$ and $10 \mathrm{mcg}$ twice daily decreased AlC significantly compared with placebo $(-0.4 \%$ and $-0.8 \%$ vs. $0.1 \%$, $P<0.001)$. Fasting plasma glucose, post-prandial plasma glucose, and body weight were all significantly decreased when both exenatide doses were compared with placebo. Nausea was the most common adverse effect; it was reported most often in the exenatide $10 \mathrm{mcg}$ group (45\%) compared with the exenatide 5 mcg group (36\%) or the placebo group (23\%), suggesting a dose-related effect. It was noted more in the first 8 weeks of therapy and declined thereafter. Hypoglycemia (5\%) was similar across all groups when exenatide was administered with metformin. This study showed that exenatide $5 \mathrm{mcg}$ and $10 \mathrm{mcg}$ subcutaneously twice daily in combination with metformin significantly decreased $\mathrm{AlC}$, fasting plasma glucose, post-prandial plasma glucose, and body weight compared with placebo in patients with type 2 diabetes.

Exenatide was also studied in 733 patients not adequately controlled with a sulfonylurea, metformin, or a combination of both in a double-blind, placebo controlled trial. ${ }^{29}$ Patients were randomized to receive placebo or exenatide $5 \mathrm{mcg}$ or $10 \mathrm{mcg}$ subcutaneously twice daily for 30 weeks. Patients had similar baseline characteristics: AlC of $8.5 \%$, mean age of 55 years, and an average BMI of $33.6 \mathrm{~kg} / \mathrm{m}^{2}$. Similar results were seen, as in the previous trials, with decreases in $\mathrm{AlC}$, fasting plasma glucose, post-prandial glucose, and body weight when exenatide $5 \mathrm{mcg}$ or $10 \mathrm{mcg}$ twice daily were compared with placebo. Nausea was again the most commonly reported adverse event, with the highest incidence seen with exenatide $10 \mathrm{mcg}(48.5 \%)$ and exenatide $5 \mathrm{mcg}(39.2 \%)$ compared with placebo (20.6\%). Tolerance to nausea developed several weeks after initiation of treatment. In this study, the incidence of hypoglycemia was 4- and 5-fold greater in both treatment groups (5 mcg: 19.2\%, $10 \mathrm{mcg}: 27.8 \%$ ) compared with the study by DeFronzo et al. (4.5\% and 5.3\%, respectively). This is likely because patients were receiving a sulfonylurea 


\section{TABLE 2 Efficacy and Safety of Exenatide for the Treatment of Type 2 Diabetes}

\begin{tabular}{|c|c|c|c|c|c|c|c|}
\hline $\begin{array}{l}\text { Authors and } \\
\text { Study Design }\end{array}$ & $\begin{array}{c}\text { Dose } \\
\text { (No. Patients) }\end{array}$ & $\begin{array}{c}\text { Study } \\
\text { Parameters }\end{array}$ & \multicolumn{4}{|c|}{ Efficacy Results } & Tolerability Results \\
\hline Buse et al. (2004)27 & Exenatide 5 mcg BID & Primary: & \multicolumn{4}{|c|}{ At week 30: } & \multirow{6}{*}{$\begin{array}{l}\text { Most common (\% for } 10 \text { mcg } \\
\text { dose vs. placebo): } \\
\text { Nausea ( } 51 \% \text { vs. } 7 \% \text { ) } \\
\text { Hypoglycemia ( } 36 \% \text { vs. } 7 \% \text { ) } \\
\text { Dizziness ( } 15 \% \text { vs. } 7 \% \text { ) } \\
\text { Feeling jittery ( } 15 \% \text { vs. } 2 \% \text { ) } \\
\text { Vomiting ( } 13 \% \text { vs. } 2 \% \text { ) } \\
\text { Diarrhea ( } 9 \% \text { vs. } 4 \% \text { ) } \\
\text { Constipation ( } 9 \% \text { vs. } 3 \%) \\
\text { Headache ( } 8 \% \text { vs. } 7 \% \text { ) } \\
\text { Increased sweating ( } 8 \% \text { vs. 1\%) }\end{array}$} \\
\hline $\mathrm{R}, \mathrm{TB}, \mathrm{PC}, \mathrm{MC}$ & $(n=125)$ & $\cdot \mathrm{A} \perp \mathrm{C}$ & & AlC change & FPG & BW change & \\
\hline \multirow{4}{*}{$\begin{array}{l}\text { Patients with type } 2 \\
\text { diabetes not controlled } \\
\text { with a sulfonylurea }\end{array}$} & \multirow{4}{*}{$\begin{array}{l}\text { Exenatide } 10 \text { mcg BID } \\
(\mathrm{n}=129) \\
\text { Placebo BID ( } \mathrm{n}=123) \\
\text { Total: } 30 \text { weeks } \\
\text { Patients continued } \\
\text { sulfonylurea therapy }\end{array}$} & \multirow{4}{*}{$\begin{array}{l}\text { Secondary: } \\
\text { - FPG } \\
\text { - Body weight }\end{array}$} & $5 \mathrm{mcg}$ & $-0.46 \%$ a & $\begin{array}{l}-0.3 \mathrm{mmol} \\
\text { per } \mathrm{L}\end{array}$ & $-0.9 \mathrm{~kg}$ & \\
\hline & & & $10 \mathrm{mcg}$ & $-0.86 \%$ a & $\begin{array}{l}-0.6 \mathrm{mmol} \\
\text { per } \mathrm{L}^{\mathrm{b}}\end{array}$ & $-1.6 \mathrm{~kg} b$ & \\
\hline & & & Placebo & $+0.12 \%$ & $\begin{array}{l}+0.4 \mathrm{mmol} \\
\text { per } \mathrm{L}\end{array}$ & $-0.6 \mathrm{~kg}$ & \\
\hline & & & \multicolumn{4}{|c|}{$\begin{array}{l}\text { a } P \leq 0.001 ; \text { b } P<0.050 \text {. } \\
\text { For subjects with baseline } \mathrm{AlC}>7 \%, 41.3 \% \text { of } \\
\text { those receiving exenatide } 10 \mathrm{mcg} \text { and } 32.6 \% \\
\text { of those receiving exenatide } 5 \mathrm{mcg} \text { reached an } \\
\text { AlC } \leq 7 \% \text {, significantly greater than those } \\
\text { receiving placebo }(8.8 \%) \text {. }\end{array}$} & \\
\hline \multirow{12}{*}{$\begin{array}{l}\text { DeFronzo et al. } \\
\text { (2005) } 28 \\
\text { R, TB, PC, MC } \\
\text { Patients with type } 2 \\
\text { diabetes not controlled } \\
\text { with maximum doses of } \\
\text { metformin ( } \geq 1,500 \mathrm{mg} \\
\text { per day) }\end{array}$} & \multirow{12}{*}{$\begin{array}{l}\text { Exenatide } 5 \text { mcg BID } \\
(\mathrm{n}=110) \\
\text { Exenatide } 10 \text { mcg BID } \\
(\mathrm{n}=113) \\
\text { Placebo }(\mathrm{n}=113) \\
\text { Total: } 30 \text { weeks } \\
\text { Patients continued } \\
\text { metformin therapy }\end{array}$} & \multirow{12}{*}{$\begin{array}{l}\text { Primary: } \\
\text { - AlC } \\
\text { Secondary: } \\
\text { - AlC } \leq 7 \% \\
\text { - FPG } \\
\text { - PPG } \\
\text { - Body weight }\end{array}$} & \multicolumn{4}{|c|}{ At week 30: } & \multirow{12}{*}{$\begin{array}{l}\text { Most common (\% for } 10 \text { mcg } \\
\text { dose vs. placebo): } \\
\text { Nausea ( } 45 \% \text { vs. } 23 \%) \\
\text { Diarrhea ( } 16 \% \text { vs. } 8 \% \text { ) } \\
\text { Vomiting ( } 12 \% \text { vs. } 4 \% \text { ) } \\
\text { Upper respiratory infection } \\
\text { (10\% vs. } 11 \% \text { ) }\end{array}$} \\
\hline & & & & $\mathrm{AlC}$ & $\mathrm{AlC}$ & & \\
\hline & & & & change & $\leq 7 \%$ & FPG & \\
\hline & & & $5 \mathrm{mcg}$ & $-0.4 \%$ a & $32 \% b$ & $\begin{array}{l}-0.4 \mathrm{mmol} \\
\text { per Ld }\end{array}$ & \\
\hline & & & $10 \mathrm{mcg}$ & $-0.8 \% a$ & $46 \%{ }^{c}$ & $-0.6 \mathrm{mmol}$ & \\
\hline & & & Placebo & $+0.1 \%$ & $13 \%$ & $\begin{array}{l}+0.8 \mathrm{mmol} \\
\text { per L }\end{array}$ & \\
\hline & & & \multicolumn{4}{|c|}{${ }^{\text {a }} P<0.001 ;$ b $P<0.010 ;{ }^{\text {c }} P<0.001 ;$ d $P<0.005$} & \\
\hline & & & & \multicolumn{2}{|c|}{$\begin{array}{c}\text { PPG } \\
\text { (average decrease } \\
\text { from baseline) }\end{array}$} & $\begin{array}{l}\text { Change } \\
\text { in body } \\
\text { weight }\end{array}$ & \\
\hline & & & $5 \mathrm{mcg}$ & \multicolumn{2}{|c|}{$34 \%$ a } & $-1.6 \mathrm{kgb}$ & \\
\hline & & & $10 \mathrm{mcg}$ & \multicolumn{2}{|c|}{$34 \%$ a } & $-2.8 \mathrm{kgc}^{\mathrm{c}}$ & \\
\hline & & & Placebo & \multicolumn{2}{|c|}{$9 \%$} & $-0.3 \mathrm{~kg}$ & \\
\hline & & & \multicolumn{4}{|c|}{${ }^{a} P=0.006 ;{ }^{b} P \leq 0.050 ;{ }^{c} P \leq 0.001$} & \\
\hline \multirow{7}{*}{$\begin{array}{l}\text { Kendall et al. (2005) } 29 \\
\text { R, DB, PC, MC } \\
\text { Patients with type } 2 \\
\text { diabetes not controlled } \\
\text { with a sulfonylurea and/ } \\
\text { or metformin therapy }\end{array}$} & \multirow{7}{*}{$\begin{array}{l}\text { Exenatide } 5 \text { mcg BID } \\
(\mathrm{n}=245) \\
\text { Exenatide } 10 \mathrm{mcg} \text { BID } \\
(\mathrm{n}=241) \\
\text { Placebo }(\mathrm{n}=247) \\
\text { Total: } 30 \text { weeks } \\
\text { Patients continued } \\
\text { existing oral regimen }\end{array}$} & \multirow{7}{*}{$\begin{array}{l}\text { Primary: } \\
\text { - AlC } \\
\text { Secondary: } \\
\text { - FPG } \\
\text { - Body weight }\end{array}$} & \multicolumn{4}{|c|}{ At week 30: } & \multirow{7}{*}{$\begin{array}{l}\text { Most common (\% for } 10 \mathrm{mcg} \\
\text { dose vs. placebo): } \\
\text { Nausea ( } 48.5 \% \text { vs. } 20.6 \%) \\
\text { Hypoglycemia ( } 27.8 \% \\
\text { vs. } 12.6 \% \text { ) } \\
\text { Upper respiratory infection } \\
\text { (17.4\% vs. } 19.4 \%) \\
\text { Diarrhea ( } 17.4 \% \text { vs. } 6.5 \%) \\
\text { Vomiting }(13.7 \% \text { vs. } 4.5 \%) \\
\text { Feeling jittery (11.6\% vs. } 6.9 \%) \\
\text { Headache ( } 7.5 \% \text { vs. } 4.9 \%)\end{array}$} \\
\hline & & & & AlC & & & \\
\hline & & & & change & & FPG & \\
\hline & & & $5 \mathrm{mcg}$ & $-0.55 \%$ & -0.5 & nmol per $L^{a}$ & \\
\hline & & & $10 \mathrm{mcg}$ & $-0.77 \%$ & -0.6 & nmol per $L^{a}$ & \\
\hline & & & Placebo & $+0.23 \%$ & +0.8 & mmol per L & \\
\hline & & & \multicolumn{4}{|c|}{$\begin{array}{l}\text { a } P<0.001 \\
\text { Both doses of exenatide resulted in a significant } \\
\text { decrease in body weight }(-1.6 \mathrm{~kg}) \text { compared with } \\
\text { placebo }(-0.9 \mathrm{~kg}) .\end{array}$} & \\
\hline
\end{tabular}

$A 1 C=$ glycosylated hemoglobin; $B I D=$ twice daily; $B W=$ body weight; $D B=$ double blind; $F P G=$ fasting plasma glucose; HOMA=homeostasis model assessment; $M C=$ multicenter; $P C=$ placebo controlled; $P P G=$ post-prandial plasma glucose; $R=$ randomized; $T B=$ triple blind .

Continued on next page.

in addition to metformin. The incidence of hypoglycemia in patients receiving placebo was also higher than that seen in the DeFronzo trial (12.6\% vs. 5.3\%). ${ }^{28}$ Other common adverse events with exenatide included feeling jittery, vomiting, diarrhea, upper respiratory infection, and headache. Despite the increased risk of hypoglycemia, this study showed that the addition of exenatide to patients not adequately controlled with their current regimen, including combination therapy, is effective and well tolerated.

Zinman et al. conducted a double-blind, placebo-controlled trial in 233 patients with type 2 diabetes who were stable on a 
Table 2 (continued): Efficacy and Safety of Exenatide for the Treatment of Type 2 Diabetes

\begin{tabular}{|c|c|c|c|c|c|c|c|}
\hline $\begin{array}{l}\text { Authors and } \\
\text { Study Design }\end{array}$ & $\begin{array}{c}\text { Dose } \\
\text { (No. Patients) } \\
\end{array}$ & $\begin{array}{c}\text { Study } \\
\text { Parameters }\end{array}$ & \multicolumn{4}{|c|}{ Efficacy Results } & Tolerability Results \\
\hline Zinman et al. (2007)30 & Exenatide $10 \mathrm{mcg}$ & Primary: & \multicolumn{4}{|l|}{ At week 16: } & \multirow{13}{*}{$\begin{array}{l}\text { Most common with exenatide } \\
\text { vs. placebo: } \\
\text { Nausea ( } 39.7 \% \text { vs. } 15.2 \%) \\
\text { Nasopharyngitis } \\
(13.2 \% \text { vs. } 8.0 \%) \\
\text { Vomiting }(13.2 \% \text { vs. } 0.9 \%) \\
\text { Hypoglycemia (10.7\% vs. } 7.1 \% \text { ) }\end{array}$} \\
\hline $\mathrm{DB}, \mathrm{PC}, \mathrm{MC}$ & twice daily $(\mathrm{n}=121)$ & & & \multicolumn{2}{|c|}{ AlC change } & FPG & \\
\hline \multirow{11}{*}{$\begin{array}{l}\text { Patients with type } 2 \\
\text { diabetes not controlled } \\
\text { with a thiazolidine- } \\
\text { dione }\end{array}$} & \multirow{11}{*}{$\begin{array}{l}\text { Placebo twice daily } \\
(\mathrm{n}=112) \\
\text { Patients continued on } \\
\text { existing oral regimen } \\
\text { Total: } 16 \text { weeks }\end{array}$} & \multirow{11}{*}{$\begin{array}{l}\text { Secondary: } \\
\text { - FPG } \\
\text { - Daily self- } \\
\text { monitored } \\
\text { glucose } \\
\text { - PPG } \\
\text { - HOMA } \beta \text {-cell } \\
\text { function } \\
\text { - Body weight }\end{array}$} & $\begin{array}{l}\text { Exenatide } \\
10 \mathrm{mcg}\end{array}$ & \multicolumn{2}{|c|}{$-0.89 \%^{a}$} & $\begin{array}{l}-1.59 \mathrm{mmol} \\
\text { per La }\end{array}$ & \\
\hline & & & Placebo & \multicolumn{2}{|c|}{$+0.09 \%$} & $+0.1 \mathrm{mmol}$ per $\mathrm{L}$ & \\
\hline & & & \multicolumn{4}{|l|}{ aP $<0.001$} & \\
\hline & & & & \multicolumn{2}{|c|}{$\begin{array}{l}\text { Self-monitored } \\
\text { glucose }\end{array}$} & PPG & \\
\hline & & & $\begin{array}{l}\text { Exenatide } \\
10 \text { mcg }\end{array}$ & \multicolumn{2}{|c|}{$\begin{array}{l}-1.85 \mathrm{mmol}^{-} \\
\text {per La }\end{array}$} & $\begin{array}{l}-1.58 \mathrm{mmol} \\
\text { per } \mathrm{L}^{\mathrm{a}}\end{array}$ & \\
\hline & & & Placebo & \multicolumn{2}{|c|}{$-0.14 \mathrm{mmol}$ per $\mathrm{L}$} & $-0.31 \mathrm{mmol}$ per $\mathrm{L}$ & \\
\hline & & & \multicolumn{4}{|l|}{ a $P<0.001$} & \\
\hline & & & & \multicolumn{2}{|c|}{ HOMA } & Body weight & \\
\hline & & & $\begin{array}{l}\text { Exenatide } \\
10 \text { mcg }\end{array}$ & \multicolumn{2}{|c|}{$+19 \% \mathrm{a}$} & $-1.75 \mathrm{kgb}$ & \\
\hline & & & Placebo & \multicolumn{2}{|c|}{$-6 \%$} & $-0.24 \mathrm{~kg}$ & \\
\hline & & & \multicolumn{4}{|c|}{${ }^{\mathrm{a}} P<0.005 ;$ b $P<0.001$} & \\
\hline \multirow{5}{*}{$\begin{array}{l}\text { Heine et al. (2005) } \\
\text { R, MC, OL } \\
\text { Patients with type } 2 \\
\text { diabetes not controlled } \\
\text { with a sulfonylurea and } \\
\text { metformin }\end{array}$} & \multirow{5}{*}{$\begin{array}{l}\text { Exenatide } 10 \text { mcg BID } \\
(\mathrm{n}=282) \\
\text { Insulin glargine } \\
\text { titrated to } \mathrm{FPG} \\
<100 \mathrm{mg} \text { per } \mathrm{dL} \\
(\mathrm{n}=267) \\
\text { Total: } 26 \text { weeks }\end{array}$} & \multirow{5}{*}{$\begin{array}{l}\text { Primary: } \\
\text { - AlC } \\
\text { Secondary: } \\
\text { - FPG } \\
\text { - Body weight }\end{array}$} & \multicolumn{4}{|c|}{ At week 26: } & \multirow{5}{*}{$\begin{array}{l}\text { Most common with exenatide } \\
\text { vs. insulin glargine: } \\
\text { Nausea }(57.1 \% \text { vs. } 8.6 \%) \\
\text { Vomiting }(17.4 \% \text { vs. } 3.7 \%) \\
\text { Headache }(8.9 \% \text { vs. } 8.6 \%) \\
\text { Diarrhea }(8.5 \% \text { vs. } 3.0 \%)\end{array}$} \\
\hline & & & & $\mathrm{AlC} \mathrm{ch}$ & lange & FPG & \\
\hline & & & Exenatide & -1.11 & & $-25.7 \mathrm{mg}$ per $\mathrm{dL}$ & \\
\hline & & & $\begin{array}{l}\text { Insulin } \\
\text { glargine }\end{array}$ & -1.11 & & $-51.5 \mathrm{mg}$ per $\mathrm{dL}^{\mathrm{a}}$ & \\
\hline & & & \multicolumn{4}{|c|}{$\begin{array}{l}\text { a } P<0.001 \\
\text { Exenatide therapy resulted in a significant loss in } \\
\text { body weight }(-2.3 \mathrm{~kg}) \text { compared with a gain with } \\
\text { insulin glargine }(+1.8 \mathrm{~kg}) \text { at week } 26(P<0.001)\end{array}$} & \\
\hline \multirow{11}{*}{$\begin{array}{l}\text { Kim et al. }(2007)^{32} \\
\text { R, DB, PC, phase } 2 \\
\text { Patients with type } 2 \\
\text { diabetes not controlled } \\
\text { with diet and/or } \\
\text { metformin }\end{array}$} & \multirow{11}{*}{$\begin{array}{l}\text { Exenatide LAR } 0.8 \mathrm{mg} \\
\text { weekly }(\mathrm{n}=16) \\
\text { Exenatide LAR } 2 \mathrm{mg} \\
\text { weekly }(\mathrm{n}=15) \\
\text { Placebo }(\mathrm{n}=14) \\
\text { Total: } 15 \text { weeks }\end{array}$} & \multirow{11}{*}{$\begin{array}{l}\text { Primary: } \\
\text { - Safety } \\
\text { - Plasma } \\
\text { concentration } \\
\text { Secondary: } \\
\text { - AlC change } \\
\text { - FPG } \\
\text { - Body weight }\end{array}$} & \multicolumn{4}{|c|}{ At week 15: } & \multirow{11}{*}{$\begin{array}{l}\text { Most common with exenatide } \\
\text { LAR } 2 \text { mg dose vs. placebo: } \\
\text { Nausea ( } 27 \% \text { vs. } 15 \%) \\
\text { Gastroenteritis ( } 13 \% \text { vs. } 0 \% \text { ) }\end{array}$} \\
\hline & & & & & $\mathrm{AlC}$ & FPG & \\
\hline & & & $\begin{array}{l}\text { Exenatide } \\
0.8 \mathrm{mg}\end{array}$ & & $-1.4 \%$ a & $-2.4 \mathrm{mmol}$ per $\mathrm{L}^{\mathrm{b}}$ & \\
\hline & & & Exenatide & AR $2 \mathrm{mg}$ & $-1.7 \%^{a}$ & $-2.2 \mathrm{mmol}$ per $\mathrm{L}^{\mathrm{b}}$ & \\
\hline & & & Placebo & & $+0.4 \%$ & $+1.0 \mathrm{mmol}$ per $\mathrm{L}$ & \\
\hline & & & \multicolumn{4}{|c|}{${ }^{\mathrm{a}} P<0.001 ;{ }^{\text {b }} P<0.001$} & \\
\hline & & & & & & Body weight & \\
\hline & & & Exenatide & AR $0.8 \mathrm{mg}$ & & no change & \\
\hline & & & Exenatide & AR $2 \mathrm{mg}$ & & $-3.8 \mathrm{~kg}^{\mathrm{a}}$ & \\
\hline & & & Placebo & & & no change & \\
\hline & & & $\begin{array}{l}\text { a } P<0.050 \\
\text { Once-week } \\
\text { similar plas } \\
\text { exenatide } 1\end{array}$ & $\begin{array}{l}\text { long-actir } \\
\text { ma concent } \\
\text { mcg after }\end{array}$ & $\begin{array}{l}\text { ng exenat } \\
\text { trations a } \\
6 \text { weeks }\end{array}$ & $\begin{array}{l}\text { tide } 2 \text { mg had } \\
\text { as once-daily } \\
\text { of treatment. }\end{array}$ & \\
\hline
\end{tabular}

$A I C=$ glycosylated hemoglobin; $B I D=$ twice daily; $B W=$ body weight $D B=$ double blind; $F P G=$ fasting plasma glucose; HOMA=homeostasis model assessment; $L A R=$ long-acting release; $M C=$ multicenter; $O L=$ open label; $P C=$ placebo controlled; $P P G=$ post-prandial plasma glucose; $R=$ randomized; $T B=$ triple blind . 
thiazolidinedione (rosiglitazone $\geq 4 \mathrm{mg}$ per day or pioglitazone $\geq 30 \mathrm{mg}$ per day), with or without metformin. ${ }^{30}$ Patients were randomized to receive either placebo or exenatide $(5 \mathrm{mcg}$ twice daily for 4 weeks, then increased to $10 \mathrm{mcg}$ twice daily for 12 weeks). Baseline characteristics were similar between groups, with a mean $\mathrm{AlC}$ of $7.9 \%$, age of 56 years, and BMI of $34 \mathrm{~kg}$ per $\mathrm{m}^{2}$. At week 16, there was a decrease in $\mathrm{AlC}$ with exenatide compared with an increase in the placebo group $(-0.89 \%$ vs. $+0.09 \%$, or a mean between-group difference of $-0.98,95 \%$ confidence interval $[\mathrm{CI}],-1.21$ to -0.74$)$. Exenatide also significantly decreased fasting plasma glucose, daily self-monitored glucose levels, post-prandial glucose, and body weight when compared with placebo. There was no difference in outcomes between patients who were receiving a thiazolidinedione alone compared with those receiving a thiazolidinedione with metformin. Not surprisingly, nausea was the most commonly reported adverse event compared with placebo (39.7\% vs. $15.2 \%$ ). Similar rates of hypoglycemia were seen in patients receiving exenatide and placebo (10.7\% vs. $7.1 \%$ ).

The efficacy of exenatide has also been compared with insulin, another agent frequently prescribed for managing type 2 diabetes. ${ }^{2}$ Heine et al. compared 26 weeks of therapy with either exenatide or insulin glargine in 549 patients who were taking maximum doses of metformin and a sulfonylurea in a randomized, openlabel trial. ${ }^{31}$ Patients were randomized to receive either exenatide ( 5 mcg twice daily for 4 weeks, then increased to $10 \mathrm{mcg}$ twice daily for the remainder of the study) or insulin glargine (initiated at 10 units/day and titrated as necessary to achieve a fasting blood glucose of $<100 \mathrm{mg}$ per $\mathrm{dL}$ ) for 26 weeks. AlC decreased comparably in both treatment groups. Insulin glargine decreased fasting plasma glucose to a greater extent than exenatide $(-51.5 \mathrm{mg}$ per $\mathrm{dL}$ vs. $-25.7 \mathrm{mg}$ per $\mathrm{dL}, P<0.001)$. Patients receiving exenatide had more constant blood glucose levels throughout the day compared with those receiving insulin glargine, an interesting finding since insulin glargine is thought to be a peakless insulin product. Nausea was reported approximately 6 times more with exenatide (57.1\%) compared with insulin glargine (8.6\%), most notably in the first few months of therapy. Patients receiving insulin glargine had an increase in body weight $(+1.8 \mathrm{~kg})$ compared with a loss seen in patients receiving exenatide $(-2.3 \mathrm{~kg})$. There were similar rates of hypoglycemia between insulin glargine and exenatide. Other common adverse events with exenatide therapy included constipation and dyspepsia.

A long-acting release (LAR) exenatide formulation is currently being studied but is not yet approved by the FDA. In a randomized, placebo-controlled, phase 2 study, Kim et al. evaluated 45 patients with type 2 diabetes managing their disease with diet plus exercise and/or metformin. ${ }^{32}$ At baseline, patients had an average $\mathrm{AlC}$ of $8.5 \%$ and weight of $106 \mathrm{~kg}$. Subjects were randomized to receive either exenatide LAR ( $0.8 \mathrm{mg}$ or $2 \mathrm{mg}$ ) or placebo as weekly subcutaneous injections for 15 weeks. At week $15, \mathrm{AlC}$ was significantly decreased in both the exenatide
LAR 0.8-mg and 2-mg groups versus placebo (-1.4\% and -1.7\% vs. $+0.4 \%$, respectively, $P<0.001)$. Fasting plasma glucose was also decreased by -2.4 mmol per L (exenatide LAR 0.8-mg group) and -2.2 mmol per L (exenatide LAR 2-mg group) compared with $+1.0 \mathrm{mmol}$ per $\mathrm{L}$ in the placebo group $(P<0.001)$. Body weight was significantly decreased in the exenatide LAR 2-mg group (-3.8 $\mathrm{kg}$ compared with no change in both the exenatide LAR 0.8-mg and placebo groups, $P<0.050$ ). The most common adverse effects reported were nausea, gastroenteritis, and hypoglycemia. This study shows the potential efficacy for a long-acting formulation of exenatide, which would be a novel treatment approach for this disease state.

Amori et al. recently (2007) described a meta-analysis evaluating the efficacy and safety of incretin therapy. ${ }^{33}$ Based on pooled analysis of the data from placebo-controlled trials, there was a significant reduction in AlC with exenatide therapy (-0.97\%; $95 \% \mathrm{CI},-1.13 \%$ to $-0.81 \%)$. Patients receiving exenatide were also more likely to achieve an AlC $<7 \%$ compared with those receiving placebo. Significant but modest weight loss occurred with exenatide therapy (weighted mean difference, $-2.37 \mathrm{~kg}$; 95\% CI, -3.95 to -0.78), which was progressive, dose-dependent, and did not plateau by week 30 . Severe hypoglycemia was rare; mild to moderate hypoglycemia occurred more often with exenatide than placebo (16\% vs. $7 \%$, respectively), particularly in patients receiving concomitant sulfonylurea therapy. Based on their analysis, the authors considered exenatide therapy to be an option for the treatment of non-pregnant adults with type 2 diabetes, particularly in patients with adequate beta-cell function who are at risk for developing hypoglycemia and who would benefit from weight loss.

\section{Safety of Exenatide}

Nausea is the most common side effect seen with exenatide therapy, with the incidence increasing with increasing dose. The incidence of nausea is about $44 \%$ with exenatide compared with $18 \%$ with placebo. Patients can expect to develop a tolerance to nausea within the first 2 months as exenatide use continues. ${ }^{20}$

Exenatide does not cause hypoglycemia. However, when studied as adjunctive therapy to sulfonylureas, the incidence of hypoglycemia was 4 to 5 times higher than when used in combination with other treatment options. For this reason, it is recommended to decrease the sulfonylurea dose by $50 \%$ when a patient initiates exenatide therapy. ${ }^{20}$ Studies have shown that it is not necessary to adjust metformin dosing due to any concern for hypoglycemia. ${ }^{25,28}$ Other potential adverse events that patients may experience include diarrhea (13\%), feeling jittery (9\%), dizziness (9\%), headache (9\%), and dyspepsia (6\%). ${ }^{20}$ The FDA recently issued a warning to health care professionals about the potential for exenatide to cause acute pancreatitis, an event seen in 30 patients through post-marketing reports. Twenty-one of these reports required hospitalization, with 5 of these having serious complications. Based on these reports, patients should 
discontinue exenatide therapy if any symptoms of pancreatitis develop..$^{34}$

To date, no studies have demonstrated major drug interactions with exenatide. Recent post-marketing reports suggest that concomitant use of warfarin and exenatide may result in an increased International Normalized Ratio (INR). ${ }^{20}$ Patients who are taking both warfarin and exenatide should be monitored for bleeding. Because exenatide decreases gastrointestinal emptying, medications that require rapid absorption (e.g., pain medications) should be taken at least 1 hour before or 2 hours after exenatide administration to ensure adequate absorption. ${ }^{20}$

Exenatide is not approved for, nor are there published trials evaluating its safety and efficacy, in pediatric patients. It is pregnancy category $C$ because it has been shown to cause reduced fetal and neonatal growth in animals. It is not known whether exenatide is excreted in human breast milk. Therefore, exenatide should be used cautiously, if at all, in nursing women. ${ }^{20}$

\section{Dosage and Administration of Exenatide}

Exenatide is available as pre-filled pens that deliver 60 doses of medication (either $5 \mathrm{mcg}$ or $10 \mathrm{mcg}$ per dose). Pre-filled pens should be kept refrigerated but should not be frozen. Unopened pens are good until the expiration date on the carton. Opened pens can be kept at room temperature and should be discarded 30 days after they are first used, even if some drug remains in the pen. ${ }^{20}$

Like insulin, exenatide is administered by subcutaneous injection. However, it is dosed in micrograms rather than units. Exenatide therapy should be initiated at $5 \mathrm{mcg}$ twice daily, administered within the 60-minute period before the 2 largest meals of the day (at least 6 hours apart). It should not be administered after a meal. If a patient is able to tolerate exenatide 5 mcg twice daily after 1 month, and additional blood glucose lowering is needed, the dose may be increased to $10 \mathrm{mcg}$ twice daily. Tolerance to nausea should develop over time. If a patient is currently receiving a sulfonylurea, the sulfonylurea dose should be decreased by $50 \%$ when exenatide is initiated, and the patient should be counseled on the signs and symptoms of hypoglycemia. No dose adjustment is needed for patients receiving concomitant metformin therapy or for elderly or hepatically impaired patients. ${ }^{20}$ No dosage adjustment is necessary in patients with mild to moderate renal impairment, but its use should be avoided in patients with severe renal disease (creatinine clearance $<30 \mathrm{~mL}$ per minute) or end-stage renal disease because clearance is significantly reduced.

\section{Summary for Exenatide}

Exenatide is a new treatment option for the management of type 2 diabetes that works by a novel mechanism of action. In shortterm trials, it has been shown to be safe and effective for patients with type 2 diabetes who are either at the maximum doses of or cannot tolerate metformin, sulfonylurea, and/or thiazolidinedione therapy and still need to decrease their AlC by at least $0.5 \%$ to
$1.0 \%$. It may also be a good choice for those patients concerned with weight gain from other antidiabetic medications or in those needing to lose weight to improve glycemic control since it has been shown to lead to weight loss. While clinical trials published to date have shown promising results, the trials primarily studied patients who were relatively healthy with no serious comorbidities. Post-marketing studies will provide a better picture of the long-term efficacy and safety profile of exenatide. While exenatide is a viable option for adjunctive therapy, it requires 2 injections daily, has a moderate effect on $\mathrm{AlC}$ relative to insulin, and is quite costly. Compliance should be closely assessed, particularly given the nausea seen early on in treatment.

\section{Pramlintide}

Pramlintide (Symlin, Amylin Pharmaceuticals) is an analog of the neuroendocrine hormone, amylin, which appears to be at least as potent as endogenous amylin.,.$^{9,35}$ It decreases post-prandial glucagon secretion, slows gastric emptying, and increases satiety. Because amylin dysfunction occurs in patients with diabetes, providing exogenous amylin could attenuate the issues of satiety and increased glucagon secretion, which affect patients with type 2 diabetes.

Pramlintide was approved by the FDA in March 2005; it is labeled to be given at meal times for type 1 and type 2 diabetes in patients who use meal-time insulin therapy and who have failed to achieve the desired glucose control despite optimal insulin therapy. ${ }^{35}$ Patients with type 2 diabetes may or may not also be receiving concurrent treatment with a sulfonylurea and/or metformin. The data evaluating the use of pramlintide for patients with type 2 diabetes are summarized below.

\section{Efficacy of Pramlintide}

There are 2 pivotal phase 3 trials evaluating the safety and efficacy of pramlintide in type 2 diabetes (Table 3). Both were randomized, placebo-controlled, double-blind trials that analyzed subjects for a total 52 weeks. ${ }^{36,37}$ Patients were already stable on insulin and may or may not have also been receiving metformin and/or a sulfonylurea. The baseline characteristics of subjects in both trials in all treatment groups were comparable (average age of 56 years, primarily white, average AlC of 9.1\%).

Ratner et al. randomized 538 patients to receive either placebo or pramlintide $30 \mathrm{mcg}, 75 \mathrm{mcg}$, or $150 \mathrm{mcg} 3$ times daily for 52 weeks. ${ }^{36}$ At 13 weeks, there was a $1 \%$ decrease in $\mathrm{AlC}$ in the pramlintide $75-\mathrm{mcg}$ and $150-\mathrm{mcg}$ groups. By week 52 , there was no significant difference between the pramlintide $75-\mathrm{mcg}$ and placebo groups. The pramlintide 150 -mcg group remained significantly better than placebo at week 52 , but the difference went from a $1.0 \%$ decrease at week 13 to a $0.6 \%$ difference at week 52 . The effect of pramlintide on body weight was significantly different than placebo for all 3 pramlintide doses and remained constant through week 52 , with the most dramatic weight loss occurring within the first month. While insulin use increased 
(TABLE 3 Efficacy and Safety of Pramlintide for the Treatment of Type 2 Diabetes

\begin{tabular}{|c|c|c|c|c|c|c|}
\hline $\begin{array}{l}\text { Authors and } \\
\text { Study Design }\end{array}$ & $\begin{array}{c}\text { Dose } \\
\text { (No. Patients) }\end{array}$ & $\begin{array}{c}\text { Study } \\
\text { Parameters }\end{array}$ & \multicolumn{3}{|c|}{ Efficacy Results } & $\begin{array}{l}\text { Tolerability } \\
\text { Results }\end{array}$ \\
\hline Ratner et al. (2002) ${ }^{36}$ & Pramlintide: & \multirow{7}{*}{$\begin{array}{l}\text { Primary: } \\
\text { - Change in AlC } \\
\text { - Change in } \\
\text { body weight } \\
\text { Secondary: } \\
\text { - Change in total } \\
\text { daily insulin }\end{array}$} & \multicolumn{3}{|l|}{ At week 52: } & \multirow{7}{*}{$\begin{array}{l}\text { Most common with pramlintide } \\
150 \text { mcg vs. placebo: } \\
\text { - Nausea ( } 22.9 \% \text { vs. } 16.9 \%) \\
\text { - Hypoglycemia ( } 64.6 \% \text { vs. } \\
70.6 \%) \\
\text { - Headache ( } 16 \% \text { vs. } 13.2 \% \text { ) } \\
\text { Highest drop-out rate was with } \\
\text { pramlintide } 150 \text { mcg due to } \\
\text { nausea. }\end{array}$} \\
\hline $\mathrm{R}, \mathrm{DB}, \mathrm{PC}, \mathrm{MC}$ & 30 mcg TID (n= 122) & & & $\mathrm{AlC}$ & Weight & \\
\hline \multirow{5}{*}{$\begin{array}{l}\text { Patients with type } 2 \\
\text { diabetes receiving } \\
\text { insulin with or without } \\
\text { an oral agent }\end{array}$} & 75 mcg TID (n= 136) & & $30 \mathrm{mcg}$ & $-0.4 \%$ & $-0.5 \mathrm{kga}$ & \\
\hline & $150 \operatorname{mcg}$ TID $(n=144)$ & & $75 \mathrm{mcg}$ & $-0.5 \%$ & $-0.5 \mathrm{kga}^{\mathrm{a}}$ & \\
\hline & Placebo TID (n=136) & & $150 \mathrm{mcg}$ & $-0.6 \%{ }^{a}$ & $-1 \mathrm{kga}$ & \\
\hline & Total: 52 weeks & & Placebo & $-0.2 \%$ & $1 \mathrm{~kg}$ & \\
\hline & & & \multicolumn{3}{|c|}{$\begin{array}{l}\text { a } P<0.010 \\
\text { - Relative increase in total daily insulin use was } \\
\text { less with pramlintide (all doses) compared with } \\
\text { placebo. } \\
\text { - There was a positive dose-response relationship } \\
\text { with increasing doses of pramlintide for mean } \\
\text { change in AlC through week } 52 \text {. }\end{array}$} & \\
\hline \multirow{11}{*}{$\begin{array}{l}\text { Hollander et al. } \\
\text { (2003) } \\
\text { R, DB, PC, MC } \\
\text { Patients with type } 2 \\
\text { diabetes receiving } \\
\text { insulin with or } \\
\text { without an oral agent }\end{array}$} & Pramlintide: & \multirow{11}{*}{$\begin{array}{l}\text { Primary: } \\
\text { - Change in AlC } \\
\text { at week } 26 \\
\text { Secondary: } \\
\text { - Change in AlC } \\
\text { at } 52 \text { weeks } \\
\text { - Percentage } \\
\text { of patients } \\
\text { achieving } \\
\text { AlC }<7 \% \\
\text { - Change in } \\
\text { body weight }\end{array}$} & \multicolumn{3}{|c|}{ At week 26: } & \multirow{11}{*}{$\begin{array}{l}\text { Most common with pramlintide } \\
120 \text { mcg dose vs. placebo: } \\
\text { - Nausea ( } 30 \% \text { vs. } 14 \%) \\
\text { - Headache ( } 17 \% \text { vs. } 8 \% \text { ) }\end{array}$} \\
\hline & 60 mcg TID* $(n=158)$ & & & $\mathrm{AlC}$ & Weight & \\
\hline & $90 \operatorname{mcg} \operatorname{BID}(\mathrm{n}=171)$ & & $90 \mathrm{mcg}$ & $-0.54 \%$ & $-0.7 \mathrm{kga}$ & \\
\hline & $120 \operatorname{mcg} \operatorname{BID}(n=166)$ & & $120 \mathrm{mcg}$ & $-0.68 \%$ a & $-1.1 \mathrm{kga}^{\mathrm{a}}$ & \\
\hline & Placebo TID (n= 161) & & Placebo & $-0.3 \%$ & $+0.25 \mathrm{~kg}$ & \\
\hline & Total: 52 weeks & & \multicolumn{3}{|l|}{ At week 52: } & \\
\hline & & & & AlC & Weight & \\
\hline & & & $90 \mathrm{mcg}$ & $-0.35 \%$ & $-0.5 \mathrm{~kg}$ & \\
\hline & & & $120 \mathrm{mcg}$ & $-0.62 \%$ a & $-1.4 \mathrm{kga}^{\mathrm{a}}$ & \\
\hline & & & Placebo & $-0.25 \%$ & $+0.7 \mathrm{~kg}$ & \\
\hline & $\begin{array}{l}\text { * } 60 \text { mcg TID group } \\
\text { results not reported }\end{array}$ & & \multicolumn{3}{|c|}{$\begin{array}{l}\text { a } P<0.050 \\
\text { - Up to } 3 \text { times more patients reached an } \\
\text { AlC }<7 \% \text { with pramlintide ( } 9.4 \% \text { with } 90 \mathrm{mcg} \\
\text { and } 12.2 \% \text { with } 120 \mathrm{mcg} \text { ) vs. placebo }(4.1 \%) \text {. }\end{array}$} & \\
\hline \multirow{5}{*}{$\begin{array}{l}\text { Riddle et al. (2007) } 38 \\
\text { R, DB, PC, MC } \\
\text { Pramlintide added } \\
\text { to basal insulin in } \\
\text { patients not adequately } \\
\text { controlled on basal } \\
\text { insulin }\end{array}$} & \multirow{5}{*}{$\begin{array}{l}\text { Pramlintide: } \\
(60-120 \text { mcg) with } \\
\text { each major meal } \\
\text { (BID or TID) }(\mathrm{n}=105) \\
\text { Placebo }(\mathrm{n}=107) \\
\text { All patients remained } \\
\text { on insulin glargine. } \\
\text { Total: } 16 \text { weeks }\end{array}$} & \multirow{5}{*}{$\begin{array}{l}\text { Primarily: } \\
\text { - AlC } \\
\text { - Composite for } \\
\text { diabetes } \\
\text { control } \\
\text { (AlC < } \% \text {, } \\
\text { body weight } \\
\text { neutral, no } \\
\text { hypoglycemia) }\end{array}$} & \multicolumn{3}{|c|}{ At week 16: } & \multirow{5}{*}{$\begin{array}{l}\text { Most common with pramlintide } \\
\text { vs. placebo: } \\
\text { - Nausea ( } 31 \% \text { vs. } 10 \%) \\
\text { - Mild/mod hypoglycemia } \\
\text { (44\% vs. } 47 \%)\end{array}$} \\
\hline & & & & $\mathrm{AlC}$ & Mean AlC & \\
\hline & & & Pramlintide & $-0.70 \%{ }^{a}$ & $7.8 \pm 0.1 \%$ & \\
\hline & & & Placebo & $-0.36 \%$ & $8.1 \pm 0.1 \%$ & \\
\hline & & & \multicolumn{3}{|c|}{$\begin{array}{l}\text { a } P<0.050 \\
\text { - No significant difference in patients achieving } \\
\text { AlC } \leq 7 \% \text { or } \geq 0.5 \% \text { decrease in AlC } \\
\text { - More patients receiving pramlintide achieved } \\
\text { their AlC goal without seeing weight gain } \\
(P<0.001) \\
\text { - Weight loss seen with pramlintide compared } \\
\text { with weight gain seen with placebo }(-1.6 \mathrm{~kg} \\
\text { vs. }+0.7 \mathrm{~kg} ; P<0.001)\end{array}$} & \\
\hline
\end{tabular}

$A 1 C=$ glycosylated hemoglobin; $B I D=$ twice daily; $B W=$ body weight $D B=$ double blind; $F P G=$ fasting plasma glucose; HOMA=homeostasis model assessment; $M C=$ multicenter; $O L=$ open label; $P C=$ placebo controlled; $P P G=$ post-prandial plasma glucose; $R=$ randomized; $T B=$ triple blind; TID $=3$ times daily.

among all groups, placebo and treatment alike, the increase in insulin dose in the pramlintide treatment groups increased $7.9 \%$ to $10.9 \%$ compared with an increase of $15 \%$ with placebo (statistical tests not done). Severe hypoglycemia that required glucagon or intravenous glucose occurred in 8 patients and was comparable in all treatment groups, pramlintide and placebo alike. An increased incidence of nausea was reported among the pramlintide 75-mcg and 150-mcg treatment groups, the majority of which 
was reported within the first 4 to 8 weeks of initiating therapy. While pramlintide $75 \mathrm{mcg} 3$ times daily was not an adequate dose to maintain a significant decrease in AlC compared with placebo, the study supported the dose of $150 \mathrm{mcg} 3$ times daily.

Hollander et al. randomized 656 patients to receive placebo or pramlintide (60 mcg 3 times daily or $90 \mathrm{mcg}$ or $120 \mathrm{mcg}$ twice daily) ${ }^{37}$ Results for the pramlintide $60 \mathrm{mcg} 3$-times daily group were not reported. Pramlintide therapy resulted in a decrease in AlC throughout the 52 weeks of the trial, with the most significant drop occurring at week 13. Only the 120-mcg twice-daily regimen proved to be significantly better than placebo at week 52. Like the trial by Ratner et al., both treatment groups experienced significant weight loss compared with placebo, which was only maintained throughout the trial for the pramlintide $120 \mathrm{mcg}$ group $(-1.4 \mathrm{~kg}$ vs. $+0.7 \mathrm{~kg}$ placebo, $P<0.050)$. There was no overall difference in severe hypoglycemia between treatment groups and placebo. There was a difference, though, when hypoglycemia was analyzed at separate time periods. The pramlintide $120 \mathrm{mcg}$ twice-daily group had a higher incidence of hypoglycemia than placebo or pramlintide $90 \mathrm{mcg}$ twice daily within the first 4 weeks of the study ( 0.9 event rate per patient year vs. 0.3 and 0.1 , respectively). After this point, all groups were similar. Nausea was more common with pramlintide than with placebo, most notably within the first 4 weeks of therapy. Beyond the first 4 weeks until the completion of the trial at 52 weeks, the incidence of nausea was similar between all groups.

The efficacy of pramlintide was also assessed in 212 patients suboptimally controlled with basal insulin (insulin glargine) with or without concomitant oral agents. ${ }^{38}$ Patients were randomized to receive pramlintide (60 mcg to $120 \mathrm{mcg}$ ) or placebo either 2 or 3 times daily, depending on their typical meal pattern (doses were given only with major meals). The primary end points were decrease in $\mathrm{AlC}$ relative to baseline and a composite of overall diabetes control (including $\mathrm{AlC}<7 \%$ or reduction by at least $0.5 \%$, body weight neutral, hypoglycemic events). Patients receiving pramlintide had a significant reduction in AlC compared with placebo $(-0.70$ vs. $-0.36, P<0.050)$, and more patients achieved their AlC goal while remaining weight neutral. Pramlintide offered the additional advantage of weight loss $(-1.6 \mathrm{~kg}$ vs. $+0.7 \mathrm{~kg}, P<0.001)$. This study suggests that pramlintide may be an option to mealtime insulin in patients who are not adequately controlled on basal insulin, with the primary advantage being its ability to result in weight loss rather than the weight gain typically seen with insulin therapy.

\section{Safety of Pramlintide}

The most common adverse effects with pramlintide therapy are gastrointestinal in nature. Nausea has been reported in $48 \%$ of patients compared with $17 \%$ of those receiving placebo. Anorexia is also higher with pramlintide therapy than with placebo ( $17 \%$ vs. $2 \%$ ). The incidence of vomiting, arthralgias, and fatigue was similar to that seen with placebo. ${ }^{35}$ Although pramlintide does not cause hypoglycemia by itself, it potentiates the effects of insulin. It is recommended that meal-time insulin be decreased by $50 \%$ when pramlintide therapy is initiated, and then the mealtime insulin dose be titrated as necessary. The product labeling includes a black-box warning for the potential increased risk for severe insulin-induced hypoglycemia within 3 hours of dosing. Hypoglycemia is more pronounced in patients with type 1 diabetes. ${ }^{35}$

Pramlintide has not been studied in patients receiving concurrent therapy with other agents that slow gastrointestinal motility or absorption. Extreme caution should be used in these patients in the event that pramlintide could alter absorption or gastrointestinal motility. Although pramlintide does not alter nutrient absorption, it does have the potential to alter absorption of oral medications that may be taken at the time of pramlintide administration. Oral medications, such as pain medications, should be administered 1 hour before or 2 hours after pramlintide. ${ }^{35}$

Overall, pramlintide was well tolerated in clinical trials. There have been, however, some potentially significant safety concerns with proper dosing. When the product was first available, it was only supplied in multidose vials with a labeled concentration of $0.6 \mathrm{mg}$ per $\mathrm{mL}$. Pramlintide is dosed in micrograms, not milligrams. To further the confusion, patient instructions indicated the volume to withdraw in units using an insulin syringe. Given that pramlintide is dosed at the same time as meal-time insulin, this could potentially have become a serious safety concern. ${ }^{39}$ Recently, the manufacturer took several steps to address these concerns. While the multidose vial is still available on the market, the product labeling on the vial now states a concentration of $600 \mathrm{mcg}$ per $\mathrm{mL}$. In addition, the product is now also available in 2 concentrations of a pen device to make dosing simpler and safer for patients. ${ }^{40}$

Pramlintide is not FDA-labeled for use in pediatric patients, but an open-label study in patients aged 12 to 18 years with type 1 diabetes has been published. ${ }^{41}$ Further research on the safety and efficacy of pramlintide in pediatric patients is needed. Pramlintide is rated pregnancy category $C$ and should only be used in pregnant women if the benefits outweigh the risks. It is not currently known if pramlintide is excreted in breast milk. ${ }^{35}$

\section{Dosage and Administration of Pramlintide}

Pramlintide is available as $5 \mathrm{~mL}$ multidose vials $(600 \mathrm{mcg}$ per $\mathrm{mL}$ solution) and disposable, multidose, pre-filled pens in 2 sizes. The $1.5 \mathrm{~mL}$ pen accommodates doses of $15 \mathrm{mcg}$ to $60 \mathrm{mcg}$, and the $2.7 \mathrm{~mL}$ pen is for doses of $60 \mathrm{mcg}$ to $120 \mathrm{mcg}$. Unopened vials and pre-filled pens should be stored in the refrigerator and should not be frozen. Opened vials and pens can be stored at room temperature or under refrigeration and should be discarded after 30 days, even if not empty. ${ }^{35}$

It is important to note that the pramlintide dosing for treating type 1 diabetes ( $15 \mathrm{mcg}$ to $30 \mathrm{mcg} 3$ times daily) is lower than 
that for type 2 diabetes. The dose of pramlintide for patients with type 2 diabetes should be initiated at $60 \mathrm{mcg}$ with each major meal that contains at least 250 calories or at least 30 grams of carbohydrate. If patients are able to tolerate the $60 \mathrm{mcg}$ dose after 1 week, the dose may be titrated up to 120 mcg 3 times daily. ${ }^{35}$ Pramlintide appears to be most efficacious when administered immediately prior to meals. ${ }^{42}$ The dose of rapid- and short-acting insulins should be decreased by $50 \%$ when initiating pramlintide therapy to reduce the potential for hypoglycemia. It is also important to note that pramlintide cannot be mixed with insulin and, therefore, must be given as a separate injection. ${ }^{35}$ Pramlintide dosing does not need to be adjusted for the elderly or for patients with renal or hepatic impairment; there are no studies in dialysis patients.

\section{Summary for Pramlintide}

Pramlintide is another new agent recently approved for the treatment of diabetes. Like exenatide, it offers a novel mechanism of action and can result in modest weight loss. While studies conducted to date have shown it to be effective as adjunctive therapy, lowering $\mathrm{AlC}$ by approximately $0.6 \%$, it must be used in patients who are receiving concomitant insulin therapy. Because it is also a subcutaneous injection, there may be compliance problems because it means 3 additional subcutaneous injections daily on top of the existing insulin regimen. Despite these limitations, pramlintide may provide an appropriate option for further glycemic control in highly compliant patients who have failed to achieve adequate glycemic control with an individualized insulin regimen since these patients are difficult to manage.

\section{Sitagliptin}

Sitagliptin (Januvia, Merck \& Co., Inc.) is a dipeptidyl peptidase-4 (DPP-4) inhibitor. While it does not act by mimicking the actions of natural neuroendocrine hormones, it is yet another new class of agents for the treatment of type 2 diabetes. Sitagliptin was approved by the FDA in October 2006 and is labeled for the treatment of type 2 diabetes as monotherapy or adjunctive therapy in combination with metformin, sulfonylureas, or a thiazolidinedione when the existing regimen no longer provides adequate blood glucose control. ${ }^{43}$ GLP-1 and glucose-dependent insulinotropic polypeptide (GIP) are incretin hormones that stimulate insulin secretion in the beta cell, may increase cell glucose sensitivity, aid in insulin synthesis, and improve betacell function. GLP-1 and GIP are degraded by DPP-4. Sitagliptin enhances the effect of these incretin hormones by decreasing their metabolism. ${ }^{43}$ Pharmacokinetic studies have shown that there was a 2- to 3-fold increase in active GLP-1 and GIP levels following an oral glucose tolerance test 2 hours after a dose of sitagliptin. ${ }^{44}$ Theoretically, by increasing the concentration of active GLP-1 and GIP, DPP-4's effects on insulin stimulation, cell glucose sensitivity, and beta-cell function should be enhanced. ${ }^{3-6}$ Another DDP-4 inhibitor, vildagliptin, was recently approved for use in Europe ${ }^{45}$ but has had delays in coming to the U.S. market due to concerns with skin, kidney, and liver toxicity. ${ }^{46,47}$

\section{Efficacy of Sitagliptin}

There are 4 key trials evaluating the safety and efficacy of sitagliptin (Table 4). Raz et al. assessed the efficacy of sitagliptin as monotherapy in 521 patients with type 2 diabetes in a double-blind, placebo-controlled trial. ${ }^{48}$ All patients had similar baseline characteristics, with an average AlC of $8.1 \%$ and a mean fasting plasma glucose (FPG) of $182.2 \mathrm{mg}$ per dL. Patients were randomized to receive placebo or sitagliptin $100 \mathrm{mg}$ or $200 \mathrm{mg}$ orally once daily for 18 weeks. Patients were either not receiving any medication for their diabetes or they were taking oral regimens that could be discontinued for the duration of the trial Those using insulin for glycemic control were excluded. AlC significantly decreased in both the sitagliptin $100 \mathrm{mg}$ and $200 \mathrm{mg}$ groups relative to placebo at week $18(-0.60 \%$ and $-0.48 \%$, respectively; $P<0.001)$. The ability of sitagliptin to lower $\mathrm{AlC}$ does not appear to be dose dependent since the $200 \mathrm{mg}$ dose did not affect any end points to a greater extent than the $100 \mathrm{mg}$ dose. In this trial, there were 2 characteristics that appeared to predict AlC response: patients earlier in their disease ( $\leq 3$ year history) and those who had a higher baseline AlC ( $\geq 9 \%)$. These subgroups each had an approximate $1 \%$ decrease in $\mathrm{AlC}$ at week 18. Sitagliptin also decreased the proinsulin:insulin ratio, indicating improved beta-cell function. There was no significant change in body weight. Side effects were comparable to those seen in patients receiving placebo, with the exception of a few uncommon reactions: nasopharyngitis, back pain, osteoarthritis, and extremity pain.

Aschner et al. conducted a double-blind, placebo-controlled, randomized study of 741 patients with an average baseline AlC of $8 \%$, who received monotherapy with placebo or sitagliptin (100 mg or $200 \mathrm{mg}$ ) for 24 weeks. ${ }^{49}$ Only $49 \%$ of patients had been taking an oral hypoglycemic agent prior to entering the study, which was discontinued upon enrollment. Sitagliptin $100 \mathrm{mg}$ and $200 \mathrm{mg}$ decreased AlC significantly better than placebo $(-0.61 \%$ and $-0.76 \%$ vs. $+0.18 \%$, respectively; $P<0.001)$. More patients achieved $\mathrm{AlC}<7 \%$ with sitagliptin $100 \mathrm{mg}$ and $200 \mathrm{mg}$ than placebo ( $41 \%$ and $45 \%$ vs. $17 \%$, respectively, $P<0.001$ ). Both doses of sitagliptin also significantly improved FPG, proinsulin: insulin ratio, 2-hour post-prandial glucose, and homeostasis model assessment (HOMA) beta-cell function compared with placebo. No significant differences in adverse effects between sitagliptin $100 \mathrm{mg}$ and placebo were reported.

Sitagliptin was evaluated as adjunctive therapy to pioglitazone in a double-blind, placebo-controlled trial evaluating 353 patients with type 2 diabetes (mean baseline AlC of 8.1\%). ${ }^{50}$ All patients also received pioglitazone $30 \mathrm{mg}$ or $45 \mathrm{mg}$ daily. They were randomized to receive placebo or sitagliptin $100 \mathrm{mg}$ once daily for 24 weeks. A significant decrease in AlC was seen with sitagliptin $100 \mathrm{mg}$ daily compared with placebo (-0.85\% vs. $-0.15 \%$, 
Managed Care Perspective on Three New Agents for Type 2 Diabetes

TABLE 4 Efficacy and Safety of Sitagliptin for the Treatment of Type 2 Diabetes

\begin{tabular}{|c|c|c|c|c|c|c|c|c|}
\hline $\begin{array}{l}\text { Authors and } \\
\text { Study Design }\end{array}$ & $\begin{array}{c}\text { Dose } \\
\text { (No. Patients) }\end{array}$ & $\begin{array}{c}\text { Study } \\
\text { Parameters }\end{array}$ & \multicolumn{5}{|c|}{ Efficacy Results } & Tolerability Results \\
\hline \multirow{11}{*}{$\begin{array}{l}\text { Raz et al. (2006) } 48 \\
\text { R, DB, PC } \\
\text { Sitagliptin used as } \\
\text { monotherapy }\end{array}$} & \multirow{11}{*}{$\begin{array}{l}\text { Sitagliptin: } \\
100 \text { mg once daily } \\
(\mathrm{n}=205) \\
200 \mathrm{mg} \text { once daily } \\
(\mathrm{n}=206) \\
\text { Placebo daily }(\mathrm{n}=110) \\
\text { Total: } 18 \text { weeks }\end{array}$} & \multirow{11}{*}{$\begin{array}{l}\text { Primary: } \\
\text { - AlC } \\
\text { Secondary: } \\
\text { - Change in FPG } \\
\text { - Change in } \\
\text { proinsulin: } \\
\text { insulin } \\
\text { - Change in } \\
\text { HOMA- } \beta \\
\text { function } \\
\text { - Change in } \\
2 \text { hr. PPG }\end{array}$} & \multicolumn{5}{|l|}{ At week 18: } & \multirow{11}{*}{$\begin{array}{l}\text { Most common with sitagliptin } \\
100 \text { mg vs. placebo: } \\
\text { - Diarrhea ( } 3.9 \% \text { vs. } 3.6 \%) \\
\text { - Abdominal pain } \\
\text { ( } 2 \% \text { vs. } 2.7 \%) \\
\text { - Nausea ( } 1 \% \text { vs. } 0 \%)\end{array}$} \\
\hline & & & & & \multirow{3}{*}{$\begin{array}{c}\mathrm{AlC} \\
-0.48 \%^{\mathrm{a}} \\
-0.36 \%^{\mathrm{a}}\end{array}$} & \multicolumn{2}{|r|}{ FPG } & \\
\hline & & & \multirow{2}{*}{\multicolumn{2}{|c|}{$\begin{array}{l}\text { Sitagliptin } 100 \mathrm{mg} \\
\text { Sitagliptin } 200 \mathrm{mg}\end{array}$}} & & \multicolumn{2}{|c|}{$-0.7 \mathrm{mmol}$ per $\mathrm{L}^{\mathrm{b}}$} & \\
\hline & & & & & & \multicolumn{2}{|c|}{$-0.6 \mathrm{mmol}$ per $\mathrm{L}^{\mathrm{a}}$} & \\
\hline & & & \multicolumn{2}{|c|}{ Placebo } & +0.1 & \multicolumn{2}{|c|}{ +0.4 mmol per $\mathrm{L}$} & \\
\hline & & & \multicolumn{5}{|c|}{$\mathrm{a} P \leq 0.001 ;$ b $P \leq 0.010$} & \\
\hline & & & & \multicolumn{2}{|c|}{$\begin{array}{l}\text { Proinsulin: } \\
\text { insulin }\end{array}$} & HOMA- $\beta$ & 2 hr. PPG & \\
\hline & & & $\begin{array}{l}\text { Sitagliptin } \\
100 \mathrm{mg}\end{array}$ & \multicolumn{2}{|c|}{$-0.05^{\mathrm{a}}$} & $12.1^{\mathrm{a}}$ & $\begin{array}{l}-2.3 \mathrm{mmol} \\
\text { per } \mathrm{L}^{\mathrm{c}}\end{array}$ & \\
\hline & & & $\begin{array}{l}\text { Sitagliptin } \\
200 \mathrm{mg}\end{array}$ & \multicolumn{2}{|c|}{-0.02} & $13.0^{\mathrm{a}}$ & $\begin{array}{l}-2.7 \mathrm{mmol} \\
\text { per } \mathrm{L}^{\mathrm{b}}\end{array}$ & \\
\hline & & & Placebo & \multicolumn{2}{|c|}{+0.07} & 1.0 & $\begin{array}{c}+0.3 \mathrm{mmol} \\
\text { per L }\end{array}$ & \\
\hline & & & \multicolumn{5}{|c|}{${ }^{\mathrm{a}} P<0.050 ;$ b $P \leq 0.001 ;{ }^{c} P \leq 0.010$} & \\
\hline Aschner et al. (2006) 49 & Sitagliptin: & Primary: & \multicolumn{5}{|c|}{ At week 24: } & \multirow{11}{*}{$\begin{array}{l}\text { Most common with sitagliptin } \\
100 \text { mg vs. placebo: } \\
\text { - Overall GI events }(16.4 \% \\
\text { vs. } 11.5 \%) \\
\text { - Diarrhea ( } 4.6 \% \text { vs. } 2.4 \%) \\
\text { - Nausea ( } 2.1 \% \text { vs. } 1.2 \%) \\
\text { - Vomiting ( } 1.3 \% \text { vs. } 1.2 \%)\end{array}$} \\
\hline $\mathrm{R}, \mathrm{DB}, \mathrm{PC}$ & \multirow{10}{*}{$\begin{array}{l}100 \mathrm{mg} \text { once daily } \\
(\mathrm{n}=238) \\
200 \mathrm{mg} \text { once daily } \\
(\mathrm{n}=250) \\
\text { Placebo daily }(\mathrm{n}=253) \\
\text { Total: } 24 \text { weeks }\end{array}$} & \multirow{10}{*}{$\begin{array}{l}\text { Secondary: } \\
\text { - Change in FPG } \\
\text { - Change in } \\
\text { proinsulin: } \\
\text { insulin } \\
\text { - Change in } \\
\text { HOMA- } \beta \\
\text { function } \\
\text { - Change in } \\
2 \text { hr. PPG }\end{array}$} & \multirow{2}{*}{\multicolumn{2}{|c|}{ Sitagliptin $100 \mathrm{mg}$}} & $\mathrm{AlC}$ & \multicolumn{2}{|r|}{ FPG } & \\
\hline \multirow{9}{*}{$\begin{array}{l}\text { Sitagliptin used as } \\
\text { monotherapy }\end{array}$} & & & & & $-0.61 \% \mathrm{a}^{\mathrm{a}}$ & \multicolumn{2}{|c|}{$-0.7 \mathrm{mmol}$ per $\mathrm{L}^{\mathrm{a}}$} & \\
\hline & & & Sitagliptin & $00 \mathrm{mg}$ & -0.7 & $5 \%$ a $-0.9 n$ & nmol per $L^{a}$ & \\
\hline & & & Placebo & & +0.18 & $3 \%+0.31$ & mmol per $\mathrm{L}$ & \\
\hline & & & a $P \leq 0.001$ & & & & & \\
\hline & & & & $\begin{array}{r}\text { Proin } \\
\text { inst }\end{array}$ & $\begin{array}{l}\text { ulin: } \\
\text { lin }\end{array}$ & HOMA- $\beta$ & 2 hr. PPG & \\
\hline & & & $\begin{array}{l}\text { Sitagliptin } \\
100 \mathrm{mg}\end{array}$ & -0. & & $13.2^{b}$ & $\begin{array}{l}-2.7 \mathrm{mmol} \\
\text { per } \mathrm{L}^{\mathrm{a}}\end{array}$ & \\
\hline & & & $\begin{array}{l}\text { Sitagliptin } \\
200 \mathrm{mg}\end{array}$ & -0.1 & & $13.1^{b}$ & $\begin{array}{l}-3.1 \mathrm{mmol}^{\mathrm{a}} \\
\text { per } \mathrm{L}^{\mathrm{a}}\end{array}$ & \\
\hline & & & Placebo & -0. & & 0.3 & $\begin{array}{l}-0.1 \mathrm{mmol} \\
\text { per L }\end{array}$ & \\
\hline & & & a $P \leq 0.001 ;$ b & $\leq 0.010$ & & & & \\
\hline
\end{tabular}

$A 1 C=$ glycosylated hemoglobin; $D B=$ double blind; $F P G=$ fasting plasma glucose; $G I=$ gastrointestinal; HOMA = homeostasis model assessment; $P C=$ placebo controlled; $P P G=$ post-prandial plasma glucose; $R=$ randomized.

Continued on next page.

$P<0.001)$. Twice as many patients in the sitagliptin group achieved an $\mathrm{AlC}<7 \%$ (45.4\% vs. $23 \%, P<0.001)$ at week 24 when compared with placebo. The proinsulin:insulin ratio also decreased in the treatment group versus the placebo group. The most common side effects with sitagliptin affected the gastrointestinal system.

Goldstein et al. conducted a 24-week, double-blind, randomized trial in 1,091 patients with type 2 diabetes comparing placebo, sitagliptin monotherapy, metformin monotherapy, and sitagliptin/metformin combination therapy ${ }^{51}$ Patients were either on previous metformin therapy or were not currently receiving any oral hypoglycemic agent at the time of enrollment. Patients were randomized to 1 of 6 treatment groups: sitagliptin $100 \mathrm{mg}$ daily, metformin $500 \mathrm{mg}$ twice daily, metformin 1,000 mg twice daily, sitagliptin $50 \mathrm{mg} / \mathrm{metformin} 500 \mathrm{mg}$ twice daily, sitagliptin $50 \mathrm{mg} / \mathrm{metformin} 1,000 \mathrm{mg}$ twice daily, or placebo. The sitagliptin/metformin combination therapy was administered as a single tablet given twice daily. AlC decreased significantly in all treatment groups when compared with placebo $(-0.66 \%$ to $-1.9 \%$ vs. placebo $+0.17 \%)$. All treatment arms demonstrated improvement compared with placebo with regard to FPG and proinsulin:insulin ratio. HOMA beta-cell function was only improved in the metformin $1,000 \mathrm{mg}$ twice-daily, sitagliptin $50 \mathrm{mg} / \mathrm{metformin} 500 \mathrm{mg}$ twice-daily, and sitagliptin $50 \mathrm{mg} / \mathrm{metformin} 1,000 \mathrm{mg}$ twice-daily groups. Both active combination groups (sitagliptin $50 \mathrm{mg} / \mathrm{metformin} 500 \mathrm{mg}$ 
Table 4 (continued): Efficacy and Safety of Sitagliptin for the Treatment of Type 2 Diabetes

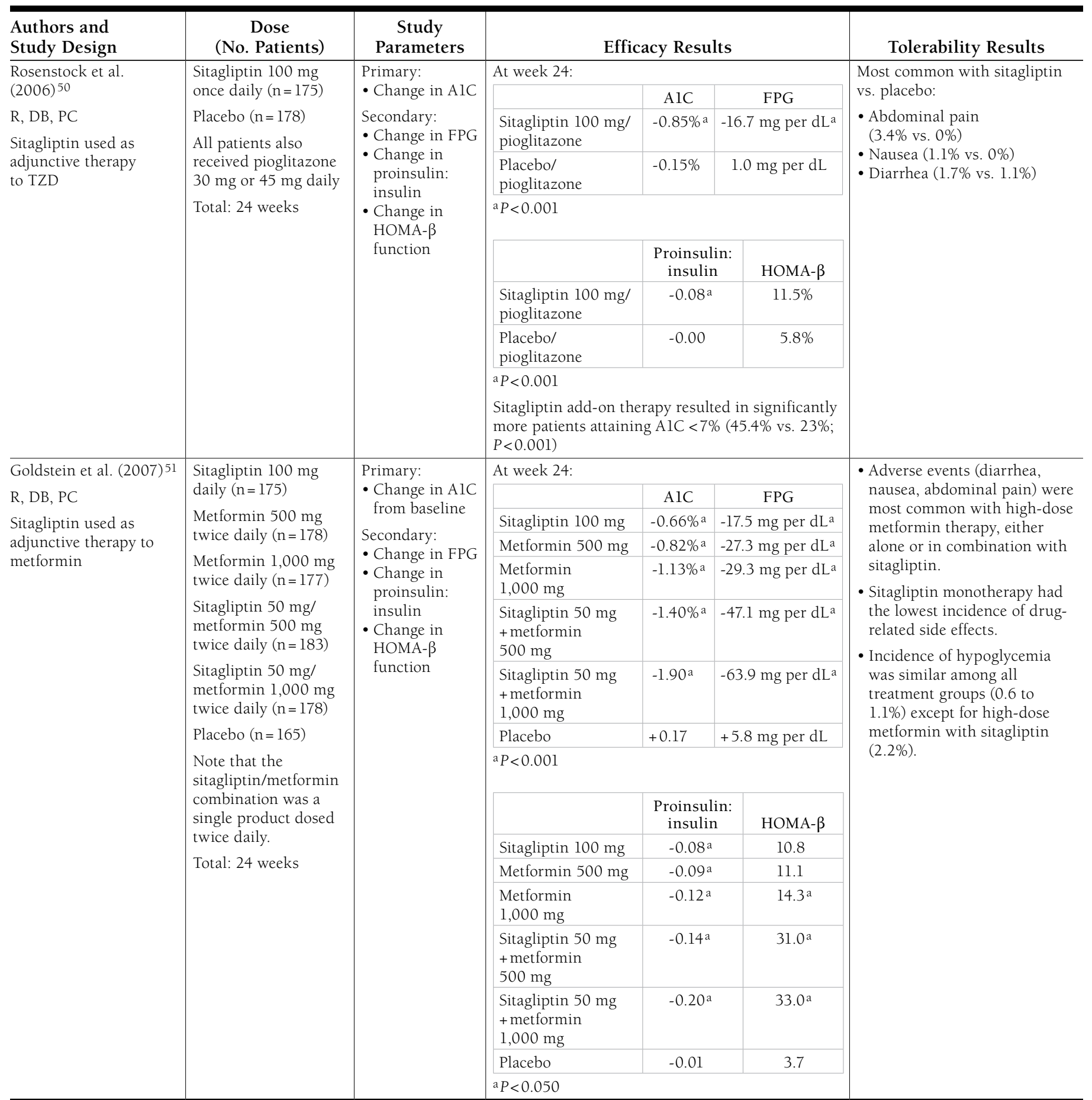

A1C=glycosylated hemoglobin; $D B=$ double blind; FPG =fasting plasma glucose; HOMA=homeostasis model assessment; $P C=$ placebo controlled; $R=$ randomized; $T Z D=$ thiazolidinedione . 
twice daily, sitagliptin $50 \mathrm{mg} / \mathrm{metformin} 1,000 \mathrm{mg}$ twice daily) decreased A1C significantly $(-1.57 \%$ and $-2.07 \%$, respectively; $P<0.001)$ when compared with sitagliptin $100 \mathrm{mg}$ daily $(-0.83 \%)$, metformin $500 \mathrm{mg}$ twice daily $(-0.99 \%)$, and metformin 1,000 mg twice daily (-1.3\%). They also decreased FPG significantly (-52.9 $\mathrm{mg}$ per $\mathrm{dL}$ and $-69.7 \mathrm{mg}$ per $\mathrm{dL}$, respectively; $P<0.001)$ when compared with sitagliptin $100 \mathrm{mg}$ daily (-23.3 mg per dL), metformin $500 \mathrm{mg}$ twice daily (-33.1 mg per dL), and metformin $1,000 \mathrm{mg}$ twice daily $(-35.1 \mathrm{mg}$ per dL). Hypoglycemia was uncommon in all treatment arms. This study showed that combination therapy with sitagliptin and metformin is more efficacious than monotherapy with either agent alone.

In a recently published meta-analysis (2007), Amori et al. performed a pooled analysis of the data from placebo-controlled trials and found a significant reduction in AlC with sitagliptin therapy $(-0.74 \% ; 95 \% \mathrm{CI},-0.85 \%$ to $-0.62 \%){ }^{33}$ Overall, the DPP-4 inhibitors were slightly less effective than other hypoglycemic agents. Patients receiving sitagliptin were also more likely to achieve an $\mathrm{AlC}<7 \%$ compared with those receiving placebo. Sitagliptin was weight neutral, a potential advantage to the weight gain seen with the sulfonylureas and thiazolidinediones. Hypoglycemia was rare, but there was an increased risk of some infections (e.g., nasopharyngitis and urinary tract infections). Based on their analysis, the authors considered sitagliptin therapy to produce moderate improvement in glycemic control, without the added benefit of weight loss seen with exenatide.

\section{Safety of Sitagliptin}

The most commonly reported adverse effects with sitagliptin are gastrointestinal in nature, specifically, abdominal pain (2.3\%), nausea (1.4\%), and diarrhea (3\%). ${ }^{43}$ Hypoglycemia has also been reported, but the incidence is not significantly higher than that reported with placebo (1.2\% vs. 0.9\%). Post-marketing experience has reported hypersensitivity reactions, including anaphylaxis, angioedema, and Stevens-Johnson syndrome. Sitagliptin was designed to be specific for the GLP substrate. However, it is not known yet whether it will have any effect on levels of the other DPP-4 substrates such as proteins involved with immunity or other hormones, which could impact its safety profile. ${ }^{19}$ At this time, no drug-drug interactions have been reported. ${ }^{43}$

Sitagliptin has not been approved for use in pediatric patients. It is rated pregnancy category $\mathrm{B}$, meaning that it is believed to be relatively safe for use in pregnancy based on studies in animals showing no fetal risk without studies available in pregnant women, or studies in animals showing a fetal risk that was not confirmed in studies conducted in pregnant women. Sitagliptin is secreted in the milk of lactating rats, but it is not known if it is secreted into human milk. Therefore, caution should be used in nursing women. ${ }^{43}$

\section{Dosage and Administration of Sitagliptin}

Sitagliptin should be dosed at $100 \mathrm{mg}$ daily with adjustments for renal insufficiency $(50 \mathrm{mg}$ for creatinine clearance $(\mathrm{CrCl}) \geq 30$ to $<50 \mathrm{~mL}$ per minute; $25 \mathrm{mg}$ for $\mathrm{CrCl}<30 \mathrm{~mL}$ per minute). No dosage adjustment is necessary for the elderly. It is administered orally and can be taken with or without food. If taken in combination with a sulfonylurea, the dose of sulfonylurea may need to be reduced to decrease the risk of hypoglycemia. ${ }^{43}$

\section{Summary of Sitagliptin}

Sitagliptin is the first drug in yet another new class of agents available for the treatment of type 2 diabetes. Unlike exenatide and pramlintide, sitagliptin offers the advantage of oral administration and is labeled for either monotherapy or adjunctive therapy with metformin, sulfonylureas, or a thiazolidinedione. It has not been studied in combination with insulin. In clinical trials, it has been shown to reduce AlC by $0.6 \%$ to $1 \%$, but it does not appear to have a positive effect on weight loss. Although the published data suggest sitagliptin to be relatively safe, it has only been tested in a limited number of patients in short-term trials. It is difficult to define the place in therapy for sitagliptin at this time because of the limited experience with it in practice, its lack of effect on weight loss, its moderate effect on lowering $\mathrm{AlC}$, and its high cost. Interestingly, despite the mild to moderate improvement seen with glycemic control and lack of long-term safety and efficacy data, $14 \%$ of new prescriptions for diabetes medications were for sitagliptin within the first 6 weeks of its approval. ${ }^{19}$

\section{Place in Therapy for These Three New Agents}

Effective management of type 2 diabetes is handled in a step-wise approach (Figure).,10,12 Diet and exercise are important for all patients. However, diet and exercise alone are often inadequate to provide sufficient, sustainable clinical benefit. The current ADA guidelines recommend initial therapy with metformin at the time of diagnosis.,10 Patients unable to tolerate metformin or in whom it is contraindicated should initiate therapy with a sulfonylurea. ${ }^{12}$ Many patients will eventually require an additional agent over time in order to maintain adequate blood glucose control. ADA guidelines recommend adding either a sulfonylurea, a thiazolidinedione, or bedtime insulin to metformin at this point. ${ }^{10}$ The choice of agent should take into consideration patient preferences, medication cost, and side-effect profile. Many patients are reluctant to initiate parenteral therapy and favor oral treatment options. For this reason, sulfonylureas are often added to metformin because they are affordable and have long-term clinical experience. ${ }^{12}$ Thiazolidinediones offer the advantage of oral administration, but they are a costly option, and their definitive role remains unclear, because there is concern with potential adverse cardiovascular effects with rosiglitazone., ${ }^{1,52-54}$ Insulin is the preferred option for patients in whom AlC is still high $(\geq 8.5 \%)$ because it remains the most potent glycemic-lowering agent available; however, it is often associated with considerable 


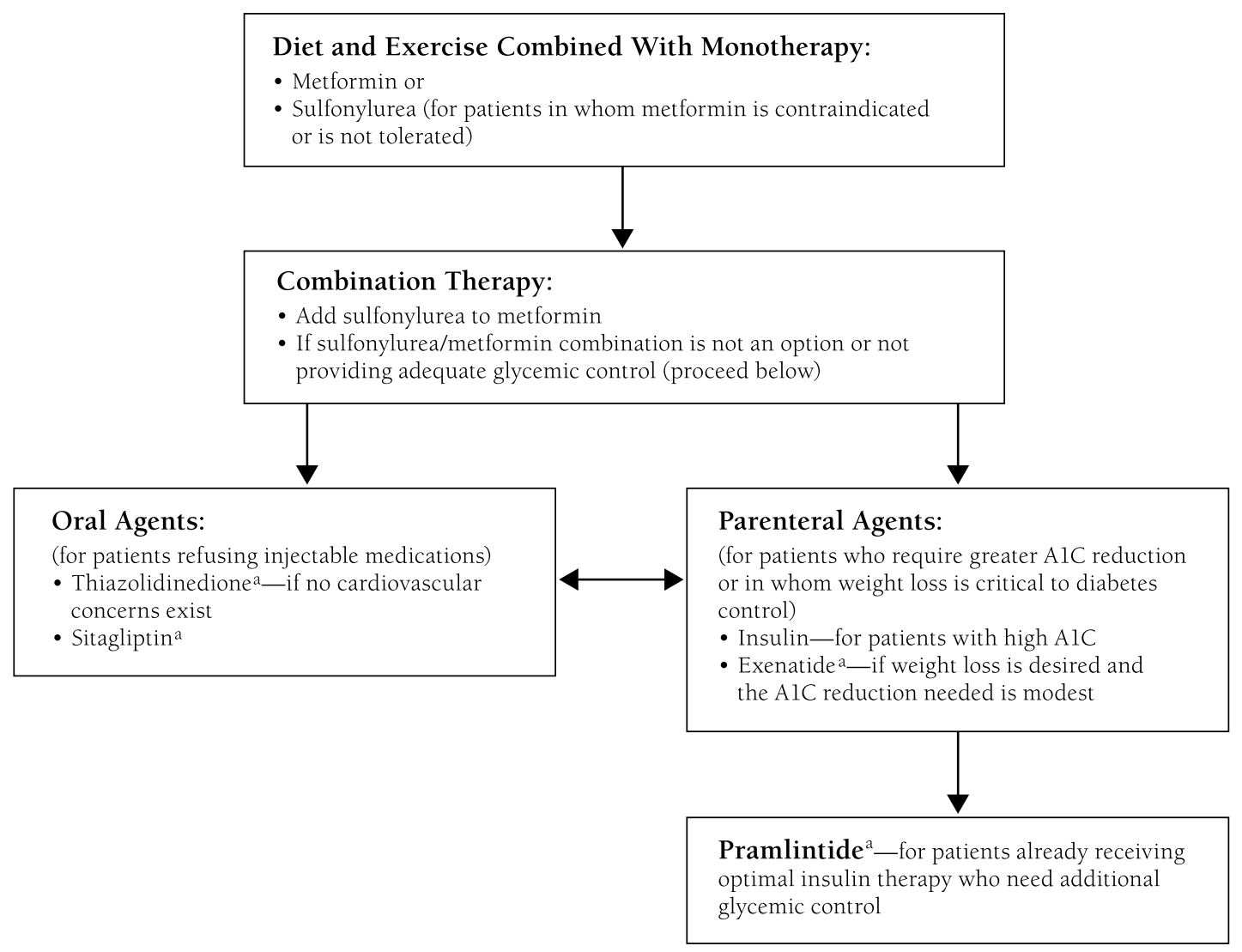

a Note: Costly compared with other treatment options.

Adapted from ADA Guidelines and modified by the authors to include the 3 new agents. 1,10,12

$A 1 C=$ glycosylated hemoglobin

weight gain. Insulin offers the advantage of being the oldest agent available, with a vast amount of clinical experience, and it is relatively affordable.

The availability of the 3 newest agents-exenatide, pramlintide, and sitagliptin — has expanded the available treatment options. To date, neither the ADA nor the AACE has defined the place in therapy for these new agents in the treatment algorithm given their relative lack of long-term safety and efficacy data. ${ }^{1,10,11}$ All 3 agents decrease $\mathrm{AlC}$ to an equivalent extent (0.5\% to $1 \%$ ), notably less than the reduction seen with the long-standing treatments: insulin, metformin, and sulfonylureas. Each agent offers a unique set of advantages and disadvantages, which must be considered when selecting therapy. Exenatide and pramlintide have a favorable effect on weight loss, which is the most attractive feature of these agents, but they must be administered by subcutaneous injection. In addition, pramlintide must be used in combination with insulin, thereby requiring multiple additional injections daily. Sitagliptin has the advantage of being an oral agent that does not cause hypoglycemia, but it is weight neutral (unlike exenatide and pramlintide). All of these new agents are considerably more costly than most of the older agents (Table 1). Because these costs are not insignificant, they should be considered when selecting an appropriate agent for any given patient.

Several factors must be considered when determining appropriate treatment options for the management of patients with type 2 diabetes. Side-effect profile (risk for hypoglycemia, history of cardiovascular disease, effect on weight), route of administration (oral vs. subcutaneous), ability to pay for the medication, and 
patient preferences are all important factors to consider so that the treatment regimen is well tolerated, affordable, and accomplishes the goals for the individual patient. Selection of the most appropriate treatment option should assess risk versus benefit. In patients who cannot tolerate or are unwilling to use agents that have established long-term safety and efficacy (i.e., insulin, metformin, sulfonylureas), a newer agent may be a reasonable treatment option.

As these new, high-cost agents for the management of diabetes have become available, managed care organizations (MCOs) are faced with the potential overuse or misuse of these agents and the associated expense. The ability of drug companies to influence prescribing through direct and indirect marketing strategies can make it difficult for physicians and patients to objectively assess the benefits and risks of the new agents. Exenatide and pramlintide have been considered by some clinicians for their ability to cause weight loss. This same benefit, though, has been viewed as a concern by many managed care providers because of the potential for misuse. Although typically covered in some manner by MCOs and Medicare Part D plans, the use of these new agents is often restricted in some way because of their high cost, limited published data, lack of data about their long-term safety and efficacy, limited clinical experience, and concerns of potential misuse for weight loss. Some plans have implemented step edits requiring a prior history of the use of older hypoglycemic agents in order to allow for coverage of the new agents..$^{56}$

MCOs can also implement electronic edits to ensure prior or coincident therapy with an antihyperglycemic agent to ensure that exenatide is being used in patients with diabetes and not solely for weight loss. In addition, quantity limits on the prescribing of these agents may be used to limit the dosing to that which has been approved by the FDA and beyond which additional benefit has not been seen (e.g., maximum $100 \mathrm{mg}$ of sitagliptin per day). In some cases, plans may require priorauthorization for the use of these new agents. Ideally, health plans can implement automated control measures that look back in the claims history to gather relevant data. Doing so is transparent to the prescriber and helps to reduce the volume of priorauthorization requests needing processing, a function that is time consuming and costly to the managed care plan. However, even with an automated system, criteria for appropriate use need to be defined for new patients or employers where no claims history exists. The control systems that managed care plans use to restrict the use of these agents will differ depending on the capabilities of their claims processing systems. While restrictions on use can be problematic for health care professionals, they force a thought process to assess appropriateness of use, particularly in light of the limited data that exist to date and the aggressive marketing of these newer agents.

\section{Conclusions}

Exenatide, pramlintide, and sitagliptin are the 3 newest agents available for the management of type 2 diabetes. While each of these new agents yields only modest glycemic effects, they provide new and differing mechanisms for managing patients with diabetes. Each agent offers a unique set of advantages and disadvantages that must be considered on a per-patient basis. The primary advantage of exenatide and pramlintide, compared with sitagliptin, is the positive, although modest, effect on weight loss. However, there is limited experience with all of these agents, and their long-term safety and efficacy remain to be determined. While these 3 new agents should generally not be used early in the treatment of most patients because of their higher cost, limited efficacy, and absence of long-term safety data, they offer treatment options for patients not adequately controlled by or who have contraindications to the use of the standard therapies.

\section{Authors}

SHAWNA VANDEKOPPEL, PharmD, is an oncology pharmacy practice resident; HAE MI CHOE, PharmD, CDE, is a clinical assistant professor of pharmacy and clinical pharmacist, ambulatory care; and BURGUNDA V. SWEET, PharmD, FASHP, is director, Drug Information Service, and a clinical associate professor of pharmacy, all at the University of Michigan Health System and College of Pharmacy. At the time of this project, Shawna VanDeKoppel was a general pharmacy practice resident at the University of Michigan Health System and College of Pharmacy.

AUTHOR CORRESPONDENCE: Burgunda V. Sweet, PharmD, FASHP, Director, Drug Information Service, University of Michigan Hospitals and Health Centers, Room B2D301, University Hospital, 1500 E. Medical Center Dr., Ann Arbor, MI 48109-5008.

Tel.: 734.936.8203; Fax: 734.936.7027; E-mail: gsweet@umich.edu

\section{DISCLOSURES}

This research was not funded, and the authors reported that there are no past, current, or anticipated financial relationships (including service on advisory boards) that might be viewed as a conflict of interest.

The authors disclose that this article includes mention of exenatide LAR that is not yet approved by the FDA.

All authors contributed approximately equally to the concept and design of this study, data collection, data interpretation, and writing and revision of the manuscript.

\section{REFERENCES}

1. American Diabetes Association. Standards of medical care in diabetes2008. Diabetes Care. 2008;30(suppl):S12-S54.

2. Genath S. Diabetes mellitus. In: Federman DD, Dale DC, et al., eds. ACP Medicine. New York: WebMD Corporation; 2004. 
3. Holst JJ, Gromada J. Role of incretin hormones in the regulation of insulin secretion in diabetic and nondiabetic humans. Am J Physiol Endocrinol Metab. 2004;287:E199-E206.

4. Zander M, Madsbad S, Madsen JL, Holst JJ. Effect of 6-week course of glucagon-like peptide 1 on glycaemic control, insulin sensitivity, and $\beta$-cell function in type 2 diabetes: a parallel-group study. Lancet. 2002;359:824-30

5. Vilsboll T, Krarup T, Madsbad S, Holst JJ. Defective amplification of the late phase insulin response to glucose by GIP in obese type II diabetic patients. Diabetologia. 2002;45:1111-19.

6. Kahn SE. The relative contributions of insulin resistance and beta-cell dysfunction to the pathophysiology of type 2 diabetes. Diabetologia. 2003; 46:3-19.

7. Young A. Amylin: physiology and pharmacology. Advances in Pharmacology. First ed. New York, NY: Elsevier; 2005.

8. Ludvik B, Thomaseth K, Nolan JJ, Clodi M, Prager R, Pacini G. Inverse relation between amylin and glucagon secretion in healthy and diabetic human subjects. Eur J Clin Invest. 2003;33:316-22.

9. Fineman M, Weyer C, Maggs DG, Strobel, Kolterman OG. The human amylin analog, pramlintide, reduces postprandial hyperglucagonemia in patients with type 2 diabetes mellitus. Horm Metab Res. 2002;34:504-08.

10. Nathan DM. Buse JB, Davidson MB, et al. Management of hyperglycemia in type 2 diabetes: a consensus algorithm for the initiation and adjustment of therapy. Diabetes Care. 2006;29:1963-72.

11. Rodbard HW, Blonde L, Braithwaite SS, et al. American Association of Clinical Endocrinologists medical guidelines for clinical practice for the management of diabetes mellitus. Endocrine Pract. 2007;13:1-66. Available at: www.aace.com/pub/pdf/guidelines/DMGuidelines2007.pdf. Accessed April 19, 2008.

12. Vijan S, Choe HM, Funnell MM, et al. Management of type 2 diabetes mellitus: University of Michigan Health System Guidelines for Clinical Care. Available at: http://cme.med.umich.edu/iCME/diabetes04/. October 2007. Accessed December 19, 2007.

13. UK Prospective Diabetes Study (UKPDS) Group. Effect of intensive blood-glucose control with metformin on complications in overweight patients with type 2 diabetes (UKPDS 34). Lancet. 1998;352:854-65.

14. Turner RC, Cull CA, Frighi V, Holman R. Glycemic control with diet, sulfonylurea, metformin, or insulin in patients with type 2 diabetes mellitus: progressive requirement for multiple therapies (UKPDS 49). JAMA. 1999;281:2005-12.

15. UK Prospective Diabetes Study (UKPDS) Group. A randomized trial of efficacy of early addition of metformin in sulphonylurea-treated non-insulin dependent diabetes (UKPDS 28). Diabetes Care. 1998;21:87-92.

16. Hanefeld M, Brunetti P, Schernthaner GH, Matthews DR, Charbonnel BH. One-year glycemic control with a sulfonylurea plus pioglitazone versus a sulfonylurea plus metformin in patients with type 2 diabetes. Diabetes Care. 2004;27(1):141-47.

17. UK Prospective Diabetes Study (UKPDS) Group. Intensive blood-glucose control with sulfonylureas or insulin compared with conventional treatment and risk of complications in patients with type 2 diabetes (UKPDS 33). Lancet. 1998;352:837-53.

18. Bolen S, Feldman L, Vassy J, et al. Systematic review: comparative effectiveness and safety of oral medications for type 2 diabetes mellitus. Ann Intern Med. 2007;147:386-99.

19. Nathan DM. Finding new treatments for diabetes-how many, how fast ... how good? N Engl J Med. 2007;356:437-40

20. Byetta [package insert]. San Diego, CA: Amylin Pharmaceuticals; February 2007.
21. Kolterman OG, Kim DD, Shen L, et al. Pharmacokinetics, pharmacodynamics, and safety of exenatide in patients with type 2 diabetes mellitus. Am J Health Syst Pharm. 2005;62:173-81.

22. Parkes DG, Pittner R, Jodka C, Smith P, Young A. Insulinotropic actions of exendin-4 and glucagon-like peptide-1 in vivo and in vitro. Metabolism. 2001;50:583-89.

23. Egan JM, Clocquet AR, Elahi D. The insulintropic effect of acute exendin- 4 administered to humans: comparison of nondiabetic state to type 2 diabetes. J Clin Endocrinol Metab. 2002;87:1282-90.

24. Chen J, Couto FM, Minn AH, Shalev A. Exenatide inhibits $\beta$-cell apoptosis by decreasing thioredoxin-interacting protein. Biochem Biophys Res Commun. 2006;346:1067-74.

25. Fineman MS, Bicsak TA, Shen LZ, et al. Effect on glycemic control of exenatide (synthetic exendin-4) additive to existing metformin and/or sulfonylurea treatment in patients with type 2 diabetes. Diabetes Care. 2003;26:2370-77.

26. Poon T, Nelson P, Shen L, et al. Exenatide improves glycemic control and reduces body weight in subjects with type 2 diabetes: a dose-ranging study. Diabetes Technol Ther. 2005;7:467-76.

27. Buse JB, Henry RR, Han J, Kim DD, Fineman MS, Baron AD. Effects of exenatide (exendin-4) on glycemic control over 30 weeks in sulfonylureatreated patients with type 2 diabetes. Diabetes Care. 2004;27:2628-35.

28. DeFronzo RA, Ratner RE, Han J, Kim DD, Fineman MS, Baron AD. Effects of exenatide (exendin-4) on glycemic control and weight over 30 weeks in metformin-treated patients with type 2 diabetes. Diabetes Care. 2005;28:1092-1100

29. Kendall DM, Riddle MC, Rosenstock J, et al. Effects of exenatide (exendin-4) on glycemic control over 30 weeks in patients with type 2 diabetes treated with metformin and a sulfonylurea. Diabetes Care. 2005; 28:1083-91.

30. Zinman B, Hoogwerf BJ, Garcia SD, et al. The effect of adding exenatide to a thiazolidinedione in suboptimally controlled type 2 diabetes. Ann Intern Med. 2007;146:477-85.

31. Heine RJ, Van Gaal LF, Johns D, Mihm MJ, Widel MH, Brodows RG. Exenatide versus insulin glargine in patients with suboptimally controlled type 2 diabetes. Ann Intern Med. 2005;143:559-69.

32. Kim D, MacConnel L, Zhuang D, et al. Effects of once-weekly dosing of a long-acting release formulation of exenatide on glucose control and body weight in subjects with type 2 diabetes. Diabetes Care. 2007;30:1487-93.

33. Amori RE, Lau J, Pittas AG. Efficacy and safety of incretin therapy in type 2 diabetes: systematic review and meta analysis. JAMA. 2007; 298:194-206. Available at: http://jama.ama-assn.org/cgi/reprint/298/ $2 / 194$ ? maxtoshow $=\& H I T S=10 \&$ hits $=10 \&$ RESULTFORMAT $=\&$ fulltext $=$ sitagliptin\&searchid=1\&FIRSTINDEX=0\&resourcetype=HWCIT. Accessed April 27, 2008.

34. U.S. Food and Drug Administration. Exenatide (marketed as Byetta) information. FDA alert 10/2007. Available at: www.fda.gov/cder/drug/ infopage/exenatide/default.htm. Accessed October 29, 2007.

35. Symlin [package insert]. San Diego, CA: Amylin Pharmaceuticals; June 2005

36. Ratner RE, Want LL, Fineman MS, et al. Adjunctive therapy with the amylin analogue pramlintide leads to a combined improvement in glycemic and weight control in insulin-treated subjects with type 2 diabetes. Diabetes Technol Ther. 2002;4:51-61.

37. Hollander PA, Levy P, Fineman MS, et al. Pramlintide as an adjunct to insulin therapy improves long-term glycemic and weight control in patients with type 2 diabetes mellitus: a one year randomized controlled trial. Diabetes Care. 2003;26:784-90. 
38. Riddle M, Frias J, Zhang B, et al. Pramlintide improved glycemic control and reduced weight in patients with type 2 diabetes using basal insulin. Diabetes Care. 2007;30:2794-99. Available at: http://care.diabetesjournals. org/cgi/content/full/30/11/2794. Accessed April 27, 2008.

39. Institute for Safe Medication Practices. Mg, mcg, units, mL: How will Symlin's safety measure up? Available at: www.ismp.org/Newsletters/ ambulatory/archives/200506_2.asp. June 2005. Accesssed June 28, 2007.

40. U.S. Food and Drug Administration. Symlin supplement approval. September 25, 2007. Available at: www.fda.gov/cder/foi/appletter/2007/ 021332s006ltr.pdf. Accessed April 13, 2008.

41. Heptulla RA, Rodriguez LM, Bomgaars L, Haymond MW. The role of amylin and glucagon in the dampening of glycemic excursions in children with type 1 diabetes. Diabetes. 2005;45:1100-07.

42. Maggs DG, Fineman M, Kornstein J, et al. Pramlintide reduces postprandial glucose excursions when added to insulin lispro in subjects with type 2 diabetes: a dose-timing study. Diabetes Metab Res Rev. 2004; 20:55-60

43. Januvia [package insert]. Whitehouse Station, NJ: Merck \& Co., Inc.; October 2007.

44. Herman GA, Bergman A, Stevens C, et al. Effect of single oral doses of sitagliptin, a dipeptidyl peptidase-4 inhibitor, on incretin and plasma glucose levels after an oral glucose tolerance test in patients with type 2 diabetes. J Clin Endocrinol Metab. 2006;91:4612-19.

45. Anonymous. UPDATE 2-EU approves Novartis diabetes drug Galvus. Thomas Reuters. February 1, 2008. Available at: www.reuters.com/article/ companyNews/idUSL0116387320080201. Accessed April 19, 2008.

46. Hughes N. 2007 FDA drug approvals: a year of flux. Nat Rev Drug Discov. 2008;7:107-09.

47. Anonymous. Vildagliptin. Wikipedia. Available at: http://en.wikipedia. org/wiki/Vildagliptin. Accessed April 19, 2008.
48. Raz I, Hanefeld M, Xu L, Caria C, Williams-Herman D, Khatami H. Efficacy and safety of the dipeptidyl peptidase-4 inhibitor sitagliptin as monotherapy in patients with type 2 diabetes mellitus. Diabetologia. 2006;49:2564-71.

49. Aschner P, Kipnes MS, Lunceford JK, Sanchez M, Mickel C, WilliamsHerman DE. Effect of the dipeptidyl peptidase-4 inhibitor sitagliptin as monotherapy on glycemic control in patients with type 2 diabetes. Diabetes Care. 2006;29:2632-37.

50. Rosenstock J, Brazg R, Andryuk PJ, Lu K, Stein P. Efficacy and safety of the dipeptidyl peptidase-4 inhibitor sitagliptin added to ongoing pioglitazone therapy in patients with type 2 diabetes: a 24-week, multicenter, randomized, double-blind, placebo-controlled, parallel-group study. Clin Ther. 2006;28:1556-68.

51. Goldstein BJ, Feinglos MN, Lunceford JK, Johnson J, Williams-Herman DE. Effect of initial combination therapy with sitagliptin, a dipeptidyl peptidase-4 inhibitor, and metformin on glycemic control in patients with type 2 diabetes. Diabetes Care. 2007;30:1979-87.

52. Lincoff AM, Wolski K, Nicholls SJ, Nissen SE. Pioglitazone and risk of cardiovascular events in patients with type 2 diabetes mellitus: a metaanalysis of randomized trials. JAMA. 2007;298:1180-88.

53. Singh S, Loke YK, Furberg CD. Long-term risk of cardiovascular events with rosiglitazone: a meta-analysis. JAMA. 2007;298:1189-95.

54. Home PD, Pocock SJ, Beck-Nielsen H, et al. Rosiglitazone evaluated for cardiovascular outcomes-an interim analysis. N Engl J Med. 2007;357:28-38

55. Drugstore.com. Available at: www.drugstore.com/. Accessed April 19, 2008.

56. The Regence Group. Medication Policy Manual. Policy no. dru120, Byetta, exenatide. May 29, 2007. Available at: http://blue.regence.com/trgmedpol/ drugs/drul20.pdf. Accessed April 27, 2008. 